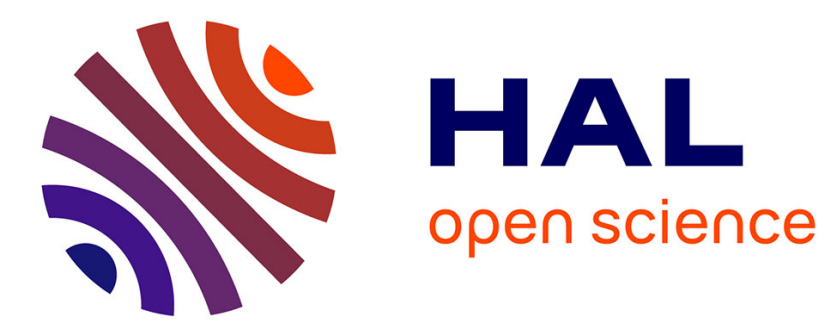

\title{
Chiral ferrocene-based P,S ligands for Ir-catalyzed hydrogenation of minimally functionalized olefins. Scope and limitations
}

Maria Biosca, Merce Coll, Florian Lagarde, Emma Bremond, Lucie

Routaboul, E. Manoury, Oscar Pamies, Rinaldo Poli, Montserrat Dieguez

\section{To cite this version:}

Maria Biosca, Merce Coll, Florian Lagarde, Emma Bremond, Lucie Routaboul, et al.. Chiral ferrocenebased P,S ligands for Ir-catalyzed hydrogenation of minimally functionalized olefins. Scope and limitations. Tetrahedron, 2016, 72 (21), pp.2623-2631. 10.1016/j.tet.2015.01.047 . hal-01929764

\section{HAL Id: hal-01929764 \\ https://hal.science/hal-01929764}

Submitted on 1 Mar 2021

HAL is a multi-disciplinary open access archive for the deposit and dissemination of scientific research documents, whether they are published or not. The documents may come from teaching and research institutions in France or abroad, or from public or private research centers.
L'archive ouverte pluridisciplinaire HAL, est destinée au dépôt et à la diffusion de documents scientifiques de niveau recherche, publiés ou non, émanant des établissements d'enseignement et de recherche français ou étrangers, des laboratoires publics ou privés. 


\title{
Chiral ferrocene-based P-S ligands for Ir-catalyzed hydrogenation of minimally functionalized olefins. Scope and limitations
}

\author{
Maria Biosca, ${ }^{a}$ Mercè Coll, ${ }^{a}$ Florian Lagarde, ${ }^{b, c}$ Emma Brémond, ${ }^{b, c}$ Lucie \\ Routaboul, ${ }^{b, c}$ Eric Manoury ${ }^{b, c^{*}}$, Oscar Pàmies, ${ }^{a}$ Rinaldo Poli, ${ }^{b, c, d}$ \\ Montserrat Diéguez, ${ }^{a, *}$
}

a Departament de Química Física i Inorgànica. Universitat Rovira i Virgili. C/ Marcel·li Domingo, s/n. 43007 Tarragona, Spain. Fax: 34-977559563; Tel: 34-977558780; email: montserrat.dieguez@urv.cat.

${ }^{\mathrm{b}}$ CNRS, LCC (Laboratoire de Chimie de Coordination), 205 route de Narbonne, BP 44099, F-31077 Toulouse Cedex 4, France CNRS. Fax: +33-561553003; Tel: +33561333174; E-mail: eric.manoury@ 1cc-toulouse.fr.

${ }^{\mathrm{c}}$ Université de Toulouse, INPT, UPS, Toulouse, France.

d Institut Universitaire de France, 103, bd Saint-Michel, 75005 Paris, France.

\begin{abstract}
A family of 12 modular ferrocenyl planar chiral phosphine-thioethers $(\mathrm{P}, \mathrm{S})$ has been studied in the asymmetric hydrogenation of minimally functionalized alkenes. These ligands differ by the substituent on sulfur or by the linker between the ferrocene moiety and the sulfur atom (no linker, methylene or methyl substituted methylene linker bearing an additional element of chirality). The cationic iridium(cod) complexes of the different P,S ligands have been efficiently synthesized. For the majority of the ligands, coordination yielded only a single diasteroisomer with full control of the absolute configuration on sulfur. The different iridium complexes have been used in the hydrogenation of various di, tri and tetrasubstituted minimally functionalized olefins. Conversions and enantioselectivities are highly dependent on the ligand and substrate structure. Full conversions and low-to-excellent enantioselectivities could be obtained
\end{abstract}


(maximum ee from 14 to $94 \%$ for 1,1-disubsituted alkenes, from 17 to $99 \%$ for trisubstituted olefins and 34\% for the tetrasubstituted alkene).

\section{Introduction}

One of the most challenging tasks of organic chemistry is the synthesis of chiral compounds, which are necessary intermediates in the preparation of a wide range of pharmaceutical, agrochemical, fine chemical and natural products. ${ }^{1} \mathrm{Up}$ to date, asymmetric hydrogenation, the atom-economical addition of $\mathrm{H}_{2}$ to a $\mathrm{C}=\mathrm{X}(\mathrm{X}=\mathrm{C}, \mathrm{N}$ or O) bond to obtain chiral compounds is one of the most efficient, sustainable and straightforward chirality-generating process. $1^{2}$ For the enantioselective hydrogenation of minimally functionalized olefins, Ir complexes with chiral P,N ligands have shown to be effective catalysts that complement the well-developed $\mathrm{Rh} / \mathrm{Ru}$ catalysts for functionalized olefins. ${ }^{3}$ Since the application of Ir-phosphine-oxazoline PHOX chiral catalysts in 1998 by Pfaltz and coworkers, ${ }^{4}$ researchers have focused on Ir-catalysts based on a wide range of P-oxazoline ligands. ${ }^{5}$ These new Ir-catalysts have significantly broadened the substrate scope. Despite the advances in Ir-based P-N catalysts, their activity and selectivity for reducing some significant minimally functionalized olefins still needs to be improved, especially since the demand for new optically active chiral centers has moved researchers into the Ir-catalyzed asymmetric reduction of more "exotic" substrates. This will require novel, highly efficient chiral ligands that are easier to handle, readily accessible, and that enhance the application range. In this respect, research has progressed to heterodonor $\mathrm{P}, \mathrm{X}$-ligands bearing more robust $\mathrm{X}$-donor groups than oxazolines (pyridines,${ }^{6}$ amides,${ }^{7}$ thiazoles,${ }^{8}$ thiazolines, ${ }^{9}$ oxazoles, ${ }^{10}$ etc.). Some of us have recently described the successful use of non-N-donor heterodonor ligands, the phosphorus-thioether ligands, for the enantioselective Ir-catalyzed reduction of minimally functionalized olefins. ${ }^{11}$ Ir-complexes modified with two families of Pthioether ligands efficiently catalyzed the hydrogenation of a large range of olefins, with results comparable to the best ones reported in the literature. Despite this success, other 
thioether-P ligands have not yet been reported and research is in progress to study the possibilities of this new class of ligands for this process.

Some of us have been involved for several years in the development of chiral ferrocene-based ligands for asymmetric catalysis. ${ }^{12}$ Ferrocene-based ligands have been successfully employed in asymmetric catalysis for more than three decades. ${ }^{13}$ They are particularly interesting because of the facile introduction of different chiralities (planar and central), their particular stereoelectronic properties and their high stability. Although they have emerged as a privileged ligand structures for asymmetric catalysis, their use in the Ir-catalyzed hydrogenation of minimally functionalized olefins has been scarce. $^{14}$

Because we are interested in more versatile and robust Ir-catalysts, we took one further step and tested ligands that incorporate the advantages of ferrocenes and the robustness of the thioether moiety. To this end, we tested a family of modular ferrocene phosphine-thioether ligands 1-12 (Figure 1) in the Ir-catalyzed hydrogenation of 34 minimally functionalized alkenes, including concrete examples with neighboring polar groups. The selection of chiral ligands contemplates systematic variations of the electronic and steric properties of the thioether moiety (ligands 1-9), ${ }^{15}$ the removal of the methylene spacer between the ferrocene and the thioether groups (ligand 10), ${ }^{16}$ as well as introducing a second stereogenic center in the methylene spacer (ligands 1112) $)^{17}$
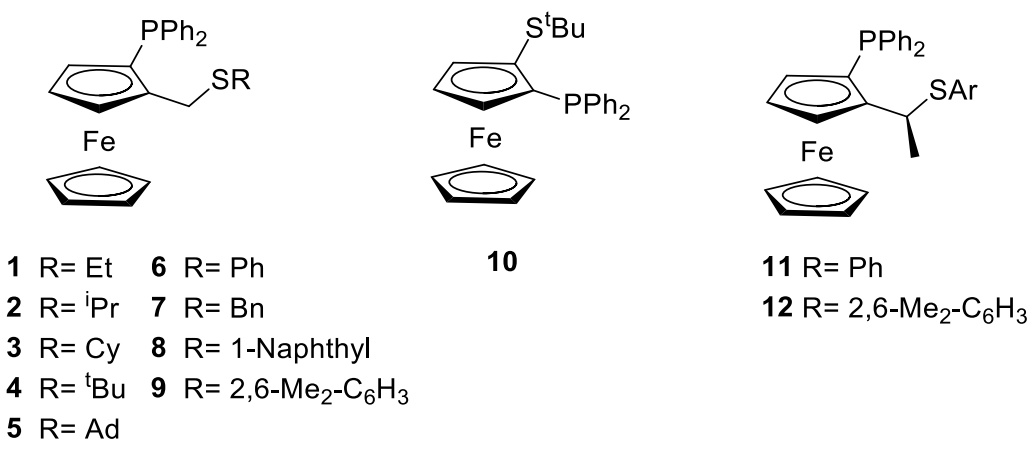

Figure 1. Ferrocene-based phosphine-thioether ligands 1-12 


\section{Results and discussion}

\section{Synthesis of Ir-catalyst precursors}

The Ir-catalyst precursors were prepared in a two-step, one-pot procedure (Scheme 1). In the first step, the appropriate ligand reacts with 0.5 equivalent of $[\operatorname{Ir}(\mu-\mathrm{Cl})(\operatorname{cod})]_{2}$ for $1 \mathrm{~h}$ at reflux. Then, $\mathrm{Cl}^{-} / \mathrm{BAr}_{\mathrm{F}}{ }^{-}$counterion exchange was achieved by a reaction with sodium tetrakis[3,5-bis(trifluoromethyl)phenyl]borate $\left(\mathrm{NaBAr}_{\mathrm{F}}\right)$ (1 equiv) in the presence of water at room temperature. The iridium catalyst precursors were isolated in pure form as air-stable orange solids in excellent yields (89-91\%).

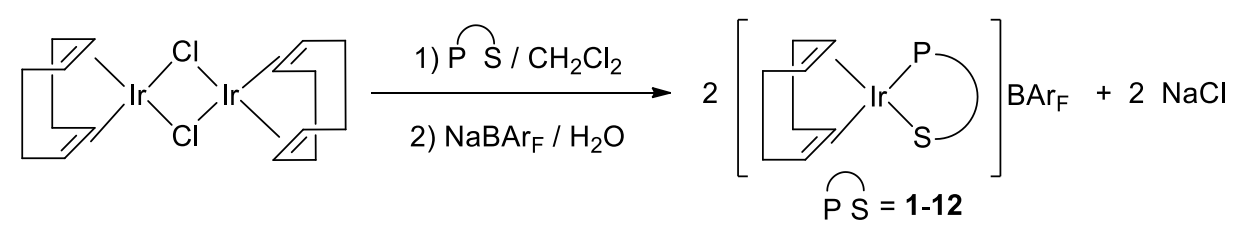

Scheme 1. Preparation of Ir-catalyst precursors $[\operatorname{Ir}(\operatorname{cod})(\mathbf{1 - 1 2})] \mathrm{BAr}_{\mathrm{F}}$

The elemental analyses were in agreement with the assigned structures. The HRMSESI spectra of $[\operatorname{Ir}(\operatorname{cod})(\mathbf{1 - 1 2})] \mathrm{BAr}_{\mathrm{F}}$ displayed the heaviest ions at $\mathrm{m} / \mathrm{z}$ which correspond to the loss of the $\mathrm{BAr}_{\mathrm{F}}$ anion from the molecular species. Crystals suitable for X-ray diffraction analysis of $[\operatorname{Ir}(\operatorname{cod})(4)] B A_{F}$ complex were also obtained in order to determine the coordination mode of the ferrocene-based phosphine-thioether ligands (Figure 2). The six-membered chelate ring adopted a boat conformation, with the thioether substituent in an equatorial position and the sulfur in an $R$ configuration as has already been observed for similar complexes. ${ }^{18}$ 


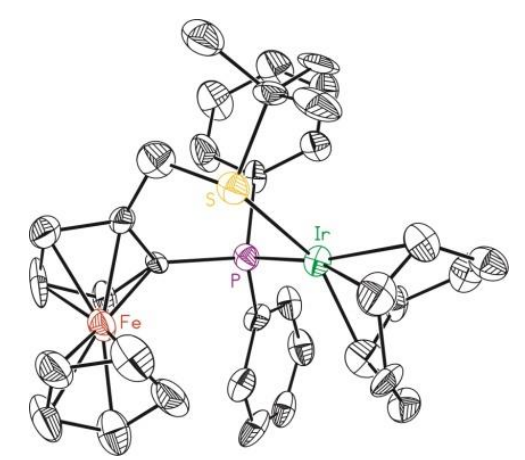

Figure 2. X-ray structure of $[\operatorname{Ir}(\operatorname{cod})(4)] \mathrm{BAr}_{F}(\mathrm{CCDC} 1033867)$ (the hydrogen atoms and $\mathrm{BAr}_{\mathrm{F}}{ }^{-}$anion have been omitted for clarity)

The ${ }^{1} \mathrm{H},{ }^{13} \mathrm{C}$, and ${ }^{31} \mathrm{P}$ NMR spectra show the expected pattern for these $C_{1}$-complexes. The VT-NMR spectra in $\mathrm{CD}_{2} \mathrm{Cl}_{2}\left(+35\right.$ to $\left.-75^{\circ} \mathrm{C}\right)$ indicate the presence of a single isomer in all cases except for $[\operatorname{Ir}(\operatorname{cod})(8)] \mathrm{BAr}_{\mathrm{F}}$ and $[\operatorname{Ir}(\operatorname{cod})(\mathbf{1 2})] \mathrm{BAr}_{\mathrm{F}}$ that were mixtures of two isomers in equilibrium at a ratio of 1:2 and 1:6, respectively. These isomers may be attributed to the two possible diastereoisomers formed when the thioether coordinates to the metal atom (note that the coordinated $\mathrm{S}$ atom is a stereogenic center), to different conformers for the six-membered chelate ring, or to both. To obtain the spatial orientation of the thioether substituent and the conformation adopted by the sixmembered chelate ring we initially performed NOESY experiments of $[\operatorname{Ir}(\operatorname{cod})(4)] \mathrm{BAr}_{\mathrm{F}}$ and $[\operatorname{Ir}(\operatorname{cod})(12)] B A r F$. Since the NOE contacts for $\operatorname{Ir} / \mathbf{4}$ were not conclusive, we studied the $[\operatorname{Ir}(\operatorname{cod})(9)] \mathrm{BAr}_{\mathrm{F}}$ analogue instead.

For complex $[\operatorname{Ir}(\operatorname{cod})(9)] \mathrm{BAr} F$ and the major isomer of $[\operatorname{Ir}(\operatorname{cod})(\mathbf{1 2})] \mathrm{BAr} F$, the NOE indicated interactions between one of the methyl groups of the thioether xylyl substituent and the phenyl group of the phosphine moiety and of that same methyl group with either the methyl substituent (for $\operatorname{Ir} / \mathbf{1 2}$ ) or one of the hydrogen atoms (for $\operatorname{Ir} / \mathbf{9}$ ) at the alkyl backbone chain (Figure 3a). In addition, the NOE indicated interactions of the other xylyl methyl group with the other hydrogen of the alkyl backbone chain and with the unsubstituted cyclopentadiene ring. These interactions can be explained assuming an equatorial disposition of the thioether group and a boat conformation of the six- 
membered chelate ring with an $R$ configuration of the sulfur atom (Figure $3 \mathrm{a}$ ), as in the $\mathrm{X}$-ray structure of $[\operatorname{Ir}(\operatorname{cod})(4)] \mathrm{BAr}_{\mathrm{F}}($ see Figure 2$)$.

(a)

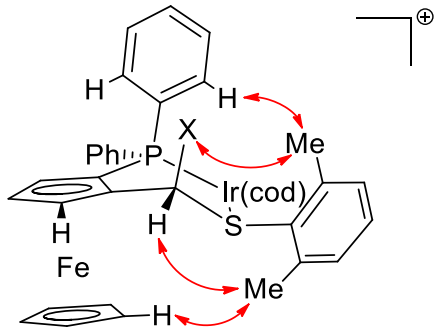

(b)

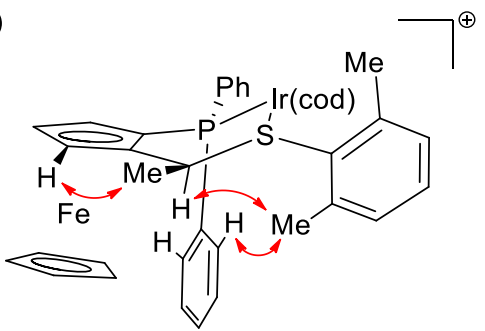

Figure 3. Main NOE contacts for (a) $[\operatorname{Ir}(\operatorname{cod})(9)] B \operatorname{Br}_{F}(X=H)$ and major isomer of $[\operatorname{Ir}(\operatorname{cod})(\mathbf{1 2})] \mathrm{BAr}_{\mathrm{F}}(\mathrm{X}=\mathrm{Me})$ and $(\mathrm{b})$ minor isomer of $[\operatorname{Ir}(\operatorname{cod})(\mathbf{1 2})] \mathrm{BAr}_{\mathrm{F}}$.

For the minor isomer of $[\operatorname{Ir}(\operatorname{cod})(\mathbf{1 2})] \mathrm{BAr} F$, we found NOE interactions between one of the xylyl methyl groups and the phenyl group of the phosphine moiety and with the hydrogen at the alkyl backbone chain (Figure 3b). We also observed NOE contacts between the methyl substituent at the alkyl backbone chain and the substituted cyclopentadiene ring. All these NOE contacts are in agreement with a boat conformation of the six-membered chelate ring and the thioether substituent in an equatorial disposition but, in contrast to previous isomers, with an $S$ configuration of the sulfur atom (Figure $3 b)$. The assignments of the isomers of $[\operatorname{Ir}(\operatorname{cod})(12)] B \mathrm{Br}_{F}$ were further confirmed by DFT studies. Figure 4 shows these calculated structures and the relative values of the formation enthalpy, being the isomer with an $R$ configuration of the sulfur atom the most stable. Complexes $[\operatorname{Ir}(\operatorname{cod})(8)] \mathrm{BAr}_{\mathrm{F}}$ and $[\operatorname{Ir}(\operatorname{cod})(\mathbf{1 2})] \mathrm{BAr} \mathrm{F}_{\mathrm{F}}$ are the first examples of incomplete control of the sulfur chirality upon coordination for ferrocenyl phosphine-thioethers with this type of backbone: only one diastereoisomer was observed for all previously reported complexes, whatever the metal or the oxidation state. ${ }^{18,19}$ 


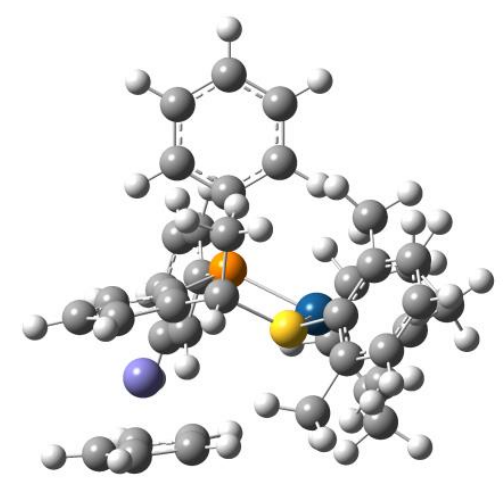

Major isomer $(0 \mathrm{KJ} / \mathrm{mol})$



Minor isomer $(11.8 \mathrm{KJ} / \mathrm{mol})$

Figure 4. Calculated structures (DFT) for cationic species of complex $[\operatorname{Ir}(\operatorname{cod})(12)] \mathrm{BAr}_{\mathrm{F}}$ and their relative formation enthalpies.

\section{Asymmetric Ir-catalyzed hydrogenation}

Asymmetric hydrogenation of minimally functionalized olefins is highly sensitive to the steric demands of the substrate.3 Unlike trisubstituted olefins, 1,1-disubstituted olefins have not been successfully hydrogenated until very recently. $3^{\mathrm{e}}$ h This is because the catalyst must control not only the face selectivity coordination (only two substituents compared with the three in trisubstituted olefins), but also the isomerization of the olefins to form the more stable $E$-trisubstituted substrates, which are hydrogenated to form the opposite enantiomer. Tetrasubstituted olefins also remain an unsolved class of substrate. $3^{\mathrm{f}, \mathrm{h}}$ The only Ir-catalysts that react with them with high yields and enantioselectivities contain the less bulky phosphanylmethyloxazoline ligands reported by Pfaltz. ${ }^{20}$ In order to evaluate the efficiency of ferrocene-based P-S ligands 1-12 in the hydrogenation of olefins with different steric demands, we initially tested them in the asymmetric reduction of the model tri-, di- and tetrasubstituted substrates S1-S3 (Table 1).

Although low-to-moderate enantioselectivities were achieved in the reduction of tetrasubstituted substrate $\mathbf{S 3}$, high enantioselectivities were obtained in the hydrogenation of model tri- and disubstituted substrates (ee's up to $85 \%$ and $82 \%$ for $\mathbf{S 1}$ and S2, respectively). The results also indicated that the ligand components need to be properly tuned for each substrate to maximize the enantioselectivities. For instance, 
while for $\mathbf{S 1}$ the best enantioselectivities were obtained with ligand $\mathbf{1 2}$ (entry 12), containing both planar and central chirality and a bulky 2,6-dimethylphenyl thioether substituent, the highest enantioselectivities for $\mathbf{S 2}$ were achieved with ligands $\mathbf{4}$ and $\mathbf{1 0}$ (entries 4 and 10), containing only planar chirality and a bulky tert-butyl thioether group. Interestingly, for disubstituted substrate $\mathbf{S 2}$ both enantiomers of the hydrogenated products could be obtained in high enantioselectivity by simply selecting the planar chirality. We also studied the reaction of model substrates $\mathbf{S 1}$ and $\mathbf{S 2}$ at low catalyst loading $(0.25 \mathrm{~mol} \%)$ using ligand 12 , and the enantioselectivities and conversion (95\% for $\mathbf{S 1}$ and $99 \%$ for $\mathbf{S 2}$ ) were maintained (entry 13).

Table 1. Ir-catalyzed hydrogenation model substrates $\mathbf{S 1 - S 3} \mathbf{3}^{\mathbf{a}}$

\begin{tabular}{|c|c|c|c|c|c|c|c|}
\hline Entry & Ligand & $\%$ Conv $^{\mathrm{b}}$ & $\% \mathrm{ee}^{\mathrm{c}}$ & $\%$ Conv $^{\mathrm{b}}$ & $\% \mathrm{ee}^{\mathrm{c}}$ & $\%$ Conv $^{\mathrm{b}}$ & $\% \mathrm{ee}^{\mathrm{c}}$ \\
\hline 1 & $\left(R_{\mathrm{p}}\right)-1$ & 100 & $25(S)$ & 100 & $22(S)$ & 75 & $9(R)$ \\
\hline 2 & $\left(R_{\mathrm{p}}\right)-2$ & 100 & 0 & 100 & $30(S)$ & $<5$ & nd \\
\hline 3 & $\left(R_{\mathrm{p}}\right)-3$ & 100 & $10(S)$ & 100 & $33(S)$ & $<5$ & nd \\
\hline 4 & $\left(R_{\mathrm{p}}\right)-4$ & 100 & $50(R)$ & 100 & $81(S)$ & 70 & $32(R)$ \\
\hline 5 & $\left(R_{\mathrm{p}}\right)-5$ & 100 & $46(S)$ & 100 & $73(S)$ & 60 & $34(R)$ \\
\hline 6 & $\left(R_{\mathrm{p}}\right)-6$ & 100 & $11(S)$ & 100 & $34(S)$ & 85 & $6(R)$ \\
\hline 7 & $\left(R_{\mathrm{p}}\right)-7$ & 100 & $30(S)$ & 100 & $16(S)$ & 35 & $8(R)$ \\
\hline 8 & $\left(R_{\mathrm{p}}\right)-\mathbf{8}$ & 100 & $24(S)$ & 100 & $32(S)$ & 65 & $6(R)$ \\
\hline 9 & $\left(R_{\mathrm{p}}\right)-9$ & 100 & $50(R)$ & 100 & $26(S)$ & 49 & $10(\mathrm{~S})$ \\
\hline 10 & $\left(R_{\mathrm{p}}\right)-\mathbf{1 0}$ & 100 & $15(S)$ & 100 & $82(R)$ & 95 & $4(R)$ \\
\hline 11 & $\left(R_{\mathrm{p}}, S\right)-\mathbf{1 1}$ & 100 & $29(S)$ & 100 & $3(R)$ & 100 & $9(R)$ \\
\hline 12 & $\left(R_{\mathrm{p}}, S\right)-\mathbf{1 2}$ & 100 & $85(S)$ & 100 & $46(R)$ & 100 & $12(\mathrm{~S})$ \\
\hline $13^{\mathrm{d}}$ & $\left(R_{\mathrm{p}}, S\right)-12$ & 95 & $84(S)$ & 99 & $46(R)$ & - & . \\
\hline
\end{tabular}

${ }^{a}$ Reactions carried out at room temperature using $0.5 \mathrm{mmol}$ of substrate and $1 \mathrm{~mol} \%$ of Ir-catalyst precursor at 100 bar of $\mathrm{H}_{2}$ (for substrates $\mathbf{S 1}$ and $\mathbf{S 3}$ ) and 1 bar (for S2) with dichloromethane (2 $\mathrm{mL}$ ) as solvent. ${ }^{\mathrm{b}}$ Conversion measured by ${ }^{1} \mathrm{H}-\mathrm{NMR}$ after $4 \mathrm{~h}$ (for $\mathbf{S 1}$ and S2) and after $18 \mathrm{~h}$ (for S3). ${ }^{\mathrm{c}}$ Enantiomeric excess determined by GC. ${ }^{\mathrm{d}}$ Reactions carried out at $0.25 \mathrm{~mol} \%$ of Ir-catalyst precursor. 
We next evaluated the new Ir/1-12 catalyst precursors in the hydrogenation of a selected range of trisubstituted substrates, most of them with neighbouring polar groups. The reduction of substrates with neighbouring polar groups has a large interest because they are relevant intermediates for the synthesis of highly valued chemicals. The most remarkable results are shown in Figure 5 (see SI for a complete set of results).



7: $100 \%$ Conv $46 \%(S)$ ee

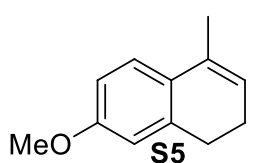

10: $100 \%$ Conv $32 \%(S)$ ee

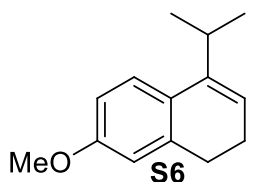

10: $100 \%$ Conv $17 \%(S)$ ee<smiles>CCOC(=O)C=C(C)c1ccc(C)cc1</smiles>

4: $100 \%$ Conv $96 \%(R)$ ee
4: $100 \%$ Conv $97 \%(R)$ ee

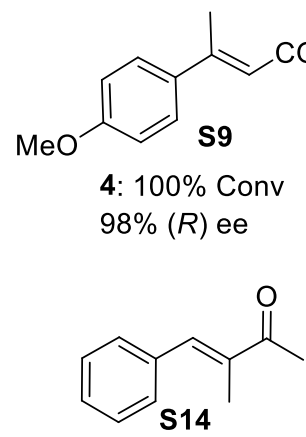

12: $100 \%$ Conv $^{a}$ $47 \%$ (S) ee<smiles>CCOC(=O)C=C(C)c1ccc(F)cc1</smiles>

4: $100 \%$ Conv 95\% $(R)$ ee

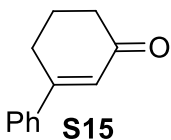

12: $100 \%$ Conv $^{a}$ $85 \%(S)$ ee<smiles>CCOC(=O)C=C(CC)c1ccccc1</smiles>

4: $100 \%$ Conv $96 \%(R)$ ee<smiles>CC1=CC(=O)CCC1</smiles>

12: $100 \%$ Conv $^{\text {a }}$ $81 \%(S)$ ee

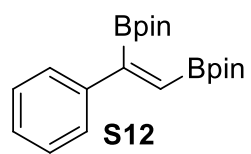

10: $100 \%$ Conv $76 \%$ (S) ee



11: $100 \%$ Conv $^{\mathrm{a}}$ $90 \%(S)$ ee

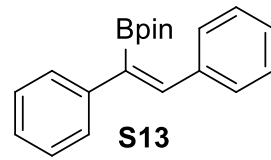

9: $100 \%$ Conv $^{\mathrm{a}}$ $64 \%(+)$ ee $84 \%(S)$ ee

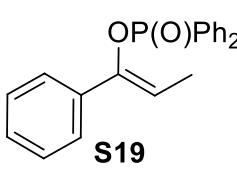

10: $100 \%$ Conv $^{\mathrm{a}}$ $92 \%(R)$ ee

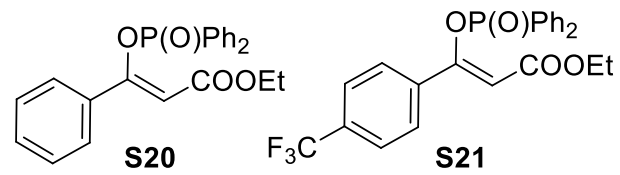

10: $82 \%$ Conv $^{a}$ $98 \%(R)$ ee
10: $76 \%$ Conv $^{\text {a }}$ $98 \%(R)$ ee



10: $100 \%$ Conv $^{\mathrm{a}}$ $93 \%(R)$ ee

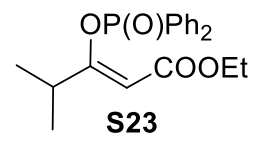

10: $54 \%$ Conv $^{\text {a }}$ $99 \%(R)$ ee

Figure 5. Selected results for the hydrogenation of trisubstituted olefins S4-S23 using $[\operatorname{Ir}(\operatorname{cod})(1-12)] B A_{F}$ catalyst precursors. Reaction conditions: 1 mol $\%$ catalyst precursor, $\mathrm{CH}_{2} \mathrm{Cl}_{2}$ as solvent, 100 bar $\mathrm{H}_{2}, 4$ h. ${ }^{a}$ Reaction carried out for $18 \mathrm{~h}$.

We first considered the reduction of aryl/alkyl substrates with Z-geometry S4-S6, which are usually hydrogenated less enantioselectively than $E$-trisubstituted olefins like S1.3 Unfortunately, as previous studies had already suggested, enantiocontrol was only moderate (ee's up to $46 \%)$. On the other hand, $[\operatorname{Ir}(\operatorname{cod})(4)] B \operatorname{Br}_{F}$ was very efficient in the reduction of several $\alpha, \beta$-unsaturated esters S7-S11. ${ }^{21}$ The ee's were between $95-98 \%$ 
and quite independent on the electronic nature of the substrate phenyl ring and on the substituent cis to the ester group. Being able to hydrogenate $\alpha, \beta$-unsaturated esters at such high ee's is of great importance because chiral carboxylic ester derivatives with tertiary benzylic aliphatic stereogenic centres are found in many fragrances, pharmaceuticals and natural products. ${ }^{22}$ This methodology represents a more sustainable route for producing these chiral carboxylic esters than other common methods such as the Co-catalyzed asymmetric conjugated reduction of $\alpha, \beta$-unsaturated esters using sodium borohydride ${ }^{23}$ and the $\mathrm{Cu}$ - and $\mathrm{Rh}$-catalyzed 1,4-reduction using very moisturesensitive hydrosilane reagents ${ }^{24}$. We then studied the reduction of alkenylboronic esters S12 and S13 which would form versatile chiral C-B bonds that can later become C-N, $\mathrm{C}-\mathrm{O}$ and $\mathrm{C}-\mathrm{C}$ bonds. The hydrogenation of alkenes containing one or two pinacolatoboron groups proceeded smoothly with enantioselectivities as high as $76 \%$. Another important class of substrates that is receiving much attention are the $\alpha, \beta$-unsaturated enones. The hydrogenation of these substrates is an elegant path for obtaining ketones with a stereogenic center in the $\alpha$ position of the carbonyl moiety. Nonetheless, they have been less studied and less successfully hydrogenated than other trisubstituted olefins. $5^{\mathrm{i}, \mathrm{u}, \mathrm{v}, 25}$ The hydrogenation of the model $\alpha, \beta$-unsaturated enone S14 proceeded with moderate enantiocontrol (ee's up to $47 \%$ ). However, it was very interesting to find that enantioselectivities increased up to $85 \%$ in the hydrogenation of more challenging cyclic enones S15 and $\mathbf{S 1 6 . 5}{ }^{\mathrm{v}}$ These latter results prompted us to focus on the hydrogenation of other difficult olefins, such as enamide $\mathbf{S 1 7},{ }^{26}$ lactone $\mathbf{S 1 8}{ }^{27}$ and enol phosphinates $\mathbf{S 1 9 - \mathbf { S 2 3 } ^ { 2 8 }}$. Very few catalytic systems can provide high enantioselectivities for these substrates so it was remarkable that we could achieve highto-excellent enantioselectivities in all of them by carefully tuning the ligand components. Thus, in the reduction of enamide S17 and lactone S18, the highest enantioselectivities (up to 90\%) were achieved using $[\operatorname{Ir}(\operatorname{cod})(\mathbf{1 1})] \mathrm{BAr}_{\mathrm{F}}$ and $[\operatorname{Ir}(\operatorname{cod})(\mathbf{1 0})] \mathrm{BAr}_{\mathrm{F}}$, respectively. $[\operatorname{Ir}(\operatorname{cod})(\mathbf{1 0})] \mathrm{BAr}_{\mathrm{F}}$ was also extremely efficient in the reduction of a range of sterically demanding enol phosphinates, including examples of pure alkyl-substituted enol phosphinates (Figure 5; S19-S23), providing comparable 
high enantioselectivities to those achieved with the best ones reported (ee's between $92-$ 99\%). The effective hydrogenation of this type of substrates opens up an appealing route for obtaining chiral organophosphinates, which can be easily transformed into high-value compounds such as alcohols and phosphines.

Then we focused our attention on extending the range of disubstituted substrates (Figure 6). Our results with several $\alpha$-alkylstyrenes bearing decreasingly sterically demanding alkyl substituents (S2, S24 and $\mathbf{S 2 5})$ indicated that enantioselectivity is affected by the nature of the alkyl chain (ee's ranging from $14 \%$ to $82 \%$ ). A plausible explanation is the competition between direct hydrogenation and isomerization. This is supported by the fact that the hydrogenation of substrate $\mathbf{S 2}$ bearing a tert-butyl group, which cannot isomerize, provides the highest enantioselectivity. We then tested a wide range of $\alpha$-tert-butylstyrene type substrates $(\mathbf{S 2 6 - S 3 2})$ to evaluate how the electronic and steric properties of the aryl group of the substrate affected the catalytic performance. The highest enantioselectivities (up to 90\%) of the series were achieved in the reduction of substrates containing electrondonating groups at the para-position of the aryl group (substrates S26 and S27).

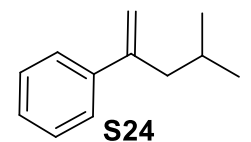

11: $100 \%$ Conv $14 \%(R)$ ee

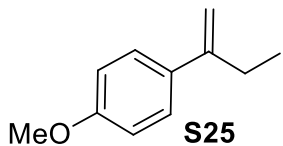

11: $100 \%$ Conv $16 \%(R)$ ee

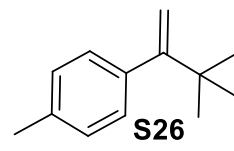

10: $100 \%$ Conv $87 \%(R)$ ee

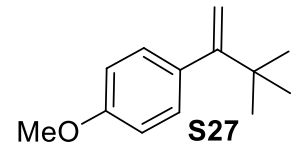

10: $100 \%$ Conv $90 \%(R)$ ee

10: $100 \%$ Conv $75 \%(R)$ ee

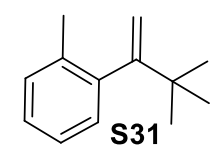

10: $100 \%$ Conv $78 \%(R)$ ee

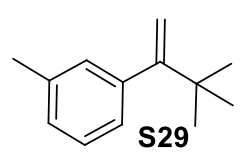

10: $100 \%$ Conv $79 \%(R)$ ee

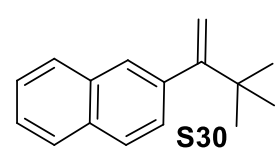

10: $100 \%$ Conv $75 \%(R)$ ee

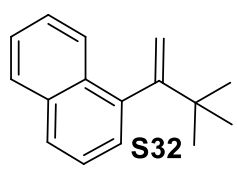

10: $100 \%$ Conv $72 \%(R)$ ee

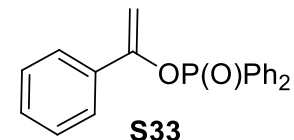

10: $100 \%$ Conv $^{a}$ $94 \%(R)$ ee

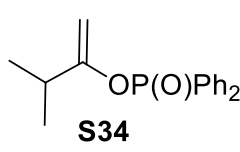

10: $100 \%$ Conv $^{a}$ $93 \%(R)$ ee 
Figure 6. Selected results for the hydrogenation of 1,1-disubstituted olefins S24-S34 using $[\operatorname{Ir}(\operatorname{cod})(1-12)] B A_{F}$ catalyst precursors. Reaction conditions: 1 mol \% catalyst precursor, $\mathrm{CH}_{2} \mathrm{Cl}_{2}$ as solvent, 1 bar $\mathrm{H}_{2}, 4$ h. ${ }^{a}$ Reactions carried out for $18 \mathrm{~h}$.

Finally, we studied whether the excellent enantioselectivities obtained in the hydrogenation of trisubstituted enol phosphinates (S19-S23, Figure 5) are maintained for the even more demanding disubstituted analogues S33 and S34. Again, $[\operatorname{Ir}(\operatorname{cod})(\mathbf{1 0})] \mathrm{BAr} F$ was able to successfully hydrogenate these substrates with excellent enantioselectivities comparable to the best ones reported. ${ }^{29}$

\section{Conclusions}

Stable cationic iridium(cod) complexes with different P,S ligands proved to be good precatalysts for the asymmetric hydrogenation of minimally functionalized olefins in terms of activities and enantioselectivities. For many substrates, the ligand fine tuning, thanks to its high modularity, enabled achieving good to excellent levels of enantioselectivity, underlining their promising potential. Therefore, these new Ircatalysts compete well with the state of art not only for model trisubstituted $\alpha, \beta$ unsaturated esters S7-S11, ${ }^{21}$ but also for demanding di- and tri-substituted enol phosphinates S19-S23 ${ }^{28}$ and S33-S34 ${ }^{29}$. In addition promising high enantioselectivities have been achieved for challenging cyclic enones $\mathbf{S 1 5}-\mathbf{S 1 6}, 5^{\mathrm{V}}$ and alkenes bearing benzyl amide ${ }^{26}(\mathbf{S 1 7})$ and $\delta$-lactone ${ }^{27}(\mathbf{S 1 8})$ groups.

\section{Experimental Section}

\section{General considerations}

All reactions were carried out using standard Schlenk techniques under an argon atmosphere. Solvents were purified and dried by standard procedures. Phosphine- 
thioether ligands $11^{17 b}$ and $12^{30}$ were prepared as previously reported. ${ }^{1} \mathrm{H},{ }^{13} \mathrm{C}$, and ${ }^{31} \mathrm{P}$ NMR spectra were recorded using a $400 \mathrm{MHz}$ or a $300 \mathrm{MHz}$ spectrometer. Chemical shifts are relative to that of $\mathrm{SiMe}_{4}\left({ }^{1} \mathrm{H}\right.$ and $\left.{ }^{13} \mathrm{C}\right)$ as internal standard or $\mathrm{H}_{3} \mathrm{PO}_{4}\left({ }^{31} \mathrm{P}\right)$ as external standard. ${ }^{1} \mathrm{H}$ and ${ }^{13} \mathrm{C}$ assignments were made on the basis of ${ }^{1} \mathrm{H}-{ }^{1} \mathrm{H}$ gCOSY and ${ }^{1} \mathrm{H}^{13} \mathrm{C}$ gHSQC. Geometries of isomers of $[\operatorname{Ir}(\operatorname{cod})(\mathbf{1 2})] \mathrm{BAr}_{\mathrm{F}}$ were optimized using the Gaussian 09 program, ${ }^{31}$ employing the $\mathrm{B}^{2} \mathrm{LYP}^{32}$ density functional and the LANL2DZ ${ }^{33}$ basis set for iridium and iron and the $6-31 G^{*}$ basis set for all other elements. ${ }^{34}$ Solvation correction was applied in the course of the optimizations using the PCM model with the default parameters of dichloromethane. ${ }^{35}$ The complexes were treated with the charge +1 and in singlet state. No symmetry constraints were applied. The energies were further refined by applying dispersion correction using the DFT-D3 ${ }^{36}$ model. All energies reported are Gibbs free energies at $298.15 \mathrm{~K}$ and calculated as $\mathrm{G}_{\text {reported }}=\mathrm{G}_{6-31 \mathrm{G}^{*}}+\mathrm{E}_{\mathrm{DFT}-\mathrm{D} 3}$

\section{General procedure for the preparation of ligands 1-9}

Ligands 1-4 and 6-7 were prepared as previously reported. ${ }^{15 a}$ The ligands $\mathbf{5}, \mathbf{8}$ and $\mathbf{9}$ were prepared using the same method from enantiomerically pure $(R)$-(2diphenylthiophosphinoferrocenyl)methanol $(100 \mathrm{mg}, \quad 0.23 \quad \mathrm{mmol})^{12 \mathrm{c}}$ and the corresponding thiol in protecting form $\mathbf{5 - S}, \mathbf{8 - S}$ and $\mathbf{9 - S}$ as thiophosphine-thioethers. The deprotected $(\mathrm{P}, \mathrm{S})$ ligands were obtained by reaction of the protected forms with $\mathrm{P}\left(\mathrm{NMe}_{2}\right)_{3}{ }^{15 \mathrm{a}}$ and immediately engaged in the coordination reaction.

5-S: Yield $131 \mathrm{mg}(97 \%) .{ }^{31} \mathrm{P}\left\{{ }^{1} \mathrm{H}\right\}$ NMR (121 MHz, $\left.\mathrm{CDCl}_{3}\right), \delta: 41.7 .{ }^{1} \mathrm{H}$ NMR $(300$ $\mathrm{MHz}, \mathrm{CDCl}_{3}$ ), $\delta: 1.63$ (m, 6H, $\left.\mathrm{CH}_{2}, \mathrm{Ad}\right), 1.75$ (m, 6H, $\left.\mathrm{CH}_{2}, \mathrm{Ad}\right), 1.97$ (m, 3H, CH, Ad), $3.72(\mathrm{~s}, 1 \mathrm{H}, \mathrm{CH}=, \mathrm{Cp}), 3.90\left(\right.$ br d, $\left.1 \mathrm{H}, \mathrm{CH}_{2},{ }^{2} J_{\mathrm{H}-\mathrm{H}}=13.0 \mathrm{~Hz}\right), 3.98\left(\right.$ br d, $1 \mathrm{H}, \mathrm{CH}_{2},{ }^{2} J_{\mathrm{H}-\mathrm{H}}$ $=13.0 \mathrm{~Hz}), 4.29$ (s, 1H, CH=, Cp), 4.36 (s, 5H, CH=, Cp), 4.66 (s, 1H, CH=, Cp), 7.55$7.35(\mathrm{~m}, 6 \mathrm{H}, \mathrm{CH}=), 7.66(\mathrm{~m}, 2 \mathrm{H}, \mathrm{CH}=), 7.86(\mathrm{~m}, 2 \mathrm{H}, \mathrm{CH}=) .{ }^{13} \mathrm{C}\left\{{ }^{1} \mathrm{H}\right\} \mathrm{NMR}(75 \mathrm{MHz}$, $\left.\mathrm{CDCl}_{3}\right), \delta: 23.7\left(\mathrm{CH}_{2}\right), 29.7(\mathrm{CH}, \mathrm{Ad}), 36.3\left(\mathrm{CH}_{2}, \mathrm{Ad}\right), 43.3\left(\mathrm{CH}_{2}, \mathrm{Ad}\right), 44.8(\mathrm{C}, \mathrm{Ad})$, $69.0\left(\mathrm{~d}, J_{\mathrm{C}-\mathrm{P}}=10.5 \mathrm{~Hz}, \mathrm{CH}=, \mathrm{Cp}\right), 70.9(\mathrm{Cp}), 73.4\left(\mathrm{~d}, J_{\mathrm{C}-\mathrm{P}}=9.3 \mathrm{~Hz}, \mathrm{CH}=, \mathrm{Cp}\right), 74.0(\mathrm{~d}$, 
$J_{\mathrm{C}-\mathrm{P}}=94.6 \mathrm{~Hz}, \mathrm{C}, \mathrm{Cp}, 74.2\left(\mathrm{~d}, J_{\mathrm{C}-\mathrm{P}}=12.7 \mathrm{~Hz}, \mathrm{CH}=, \mathrm{Cp}\right), 90.2\left(\mathrm{~d}, J_{\mathrm{C}-\mathrm{P}}=12.0 \mathrm{~Hz}, \mathrm{C}\right.$, $\mathrm{Cp}), 128.0\left(\mathrm{~d}, J_{\mathrm{C}-\mathrm{P}}=12.4 \mathrm{~Hz}, \mathrm{CH}=\right), 128.1\left(\mathrm{~d}, J_{\mathrm{C}-\mathrm{P}}=12.5 \mathrm{~Hz}, \mathrm{CH}=\right), 131.1\left(\mathrm{~d}, J_{\mathrm{C}-\mathrm{P}}=3.0\right.$ $\mathrm{Hz}, \mathrm{CH}=), 131.2\left(\mathrm{~d}, J_{\mathrm{C}-\mathrm{P}}=3.0 \mathrm{~Hz}, \mathrm{CH}=\right), 132.1\left(\mathrm{~d}, J_{\mathrm{C}-\mathrm{P}}=10.6 \mathrm{~Hz}, \mathrm{CH}=\right), 132.3\left(\mathrm{~d}, J_{\mathrm{C}-\mathrm{P}}\right.$ $=10.6 \mathrm{~Hz}, \mathrm{CH}=), 133.6\left(\mathrm{~d}, J_{\mathrm{C}-\mathrm{P}}=86.0 \mathrm{~Hz}, \mathrm{C}\right), 134.6\left(\mathrm{~d}, J_{\mathrm{C}-\mathrm{P}}=87.1 \mathrm{~Hz}, \mathrm{C}\right) . \mathrm{HR} / \mathrm{MS}$ (ESI) $m / e: 582.1273$ (M, $100 \%$; calculated for $\left.\mathrm{C}_{33} \mathrm{H}_{35} \mathrm{PS}_{2} \mathrm{Fe}: 582.1267\right)$.

5: Yield $114 \mathrm{mg}(92 \%) .{ }^{31} \mathrm{P}$ NMR (162 $\left.\mathrm{MHz}, \mathrm{CDCl}_{3}\right), \delta:-23.5 .{ }^{1} \mathrm{H}$ NMR (400 MHz, $\mathrm{CDCl}_{3}$ ), $\delta: 1.64\left(\mathrm{~m}, 6 \mathrm{H}, \mathrm{CH}_{2}, \mathrm{Ad}\right), 1.77$ (m, 6H, $\left.\mathrm{CH}_{2}, \mathrm{Ad}\right), 1.98$ (m, 3H, $\left.\mathrm{CH}, \mathrm{Ad}\right), 3.62$ $\left(\mathrm{dd}, 1 \mathrm{H}, \mathrm{CH}_{2},{ }^{2} J_{\mathrm{H}-\mathrm{H}}=13.2 \mathrm{~Hz}, J_{\mathrm{H}-\mathrm{P}}=2.4 \mathrm{~Hz}\right), 3.73(\mathrm{~b}, 1 \mathrm{H}, \mathrm{CH}=, \mathrm{Cp}), 3.75\left(\mathrm{~d}, 1 \mathrm{H}, \mathrm{CH}_{2}\right.$, $\left.{ }^{2} J_{\mathrm{H}-\mathrm{H}}=13.2 \mathrm{~Hz}\right), 4.02(\mathrm{~s}, 5 \mathrm{H}, \mathrm{CH}=, \mathrm{Cp}), 4.26(\mathrm{~m}, 1 \mathrm{H}, \mathrm{CH}=, \mathrm{Cp}), 4.55(\mathrm{~m}, 1 \mathrm{H}, \mathrm{CH}=$, $\mathrm{Cp}), 7.1-7.3(\mathrm{~m}, 5 \mathrm{H}, \mathrm{CH}=), 7.40(\mathrm{~m}, 3 \mathrm{H}, \mathrm{CH}=), 7.57(\mathrm{~m}, 2 \mathrm{H}, \mathrm{CH}=) .{ }^{13} \mathrm{C}\left\{{ }^{1} \mathrm{H}\right\} \mathrm{NMR}(100$ $\left.\mathrm{MHz}, \mathrm{CDCl}_{3}\right), \delta: 24.5\left(\mathrm{~d}, J_{\mathrm{C}-\mathrm{P}}=12.2 \mathrm{~Hz}, \mathrm{CH}_{2}\right), 29.8(\mathrm{CH}, \mathrm{Ad}), 36.5\left(\mathrm{CH}_{2}, \mathrm{Ad}\right), 43.4$ $\left(\mathrm{CH}_{2}, \mathrm{Ad}\right), 44.9(\mathrm{C}, \mathrm{Ad}), 69.5(\mathrm{CH}=, \mathrm{Cp}), 70.0(\mathrm{CH}=, \mathrm{Cp}), 71.1\left(\mathrm{~d}, J_{\mathrm{C}-\mathrm{P}}=3.8 \mathrm{~Hz}, \mathrm{CH}=\right.$, Cp), $71.5\left(\mathrm{~d}, J_{\mathrm{C}-\mathrm{P}}=3.8 \mathrm{~Hz}, \mathrm{C}, \mathrm{Cp},\right) 75.7\left(\mathrm{~d}, J_{\mathrm{C}-\mathrm{P}}=10.2 \mathrm{~Hz}, \mathrm{CH}=, \mathrm{Cp}\right), 91.3\left(\mathrm{~d}, J_{\mathrm{C}-\mathrm{P}}=\right.$ $25.1 \mathrm{~Hz}, \mathrm{C}, \mathrm{Cp}), 125.4(\mathrm{C}), 127.8(\mathrm{CH}=), 128.0\left(\mathrm{~d}, J_{\mathrm{C}-\mathrm{P}}=6.0 \mathrm{~Hz}, \mathrm{CH}=\right), 128.3$ (d, $J_{\mathrm{C}-\mathrm{P}}=$ 7.6 Hz, CH=), $128.4(\mathrm{C}), 129.2\left(\mathrm{~d}, J_{\mathrm{C}-\mathrm{P}}=6.2 \mathrm{~Hz}, \mathrm{CH}=\right), 132.6$ (d, $\left.J_{\mathrm{C}-\mathrm{P}}=17.5 \mathrm{~Hz}, \mathrm{CH}=\right)$, $135.3\left(\mathrm{~d}, J_{\mathrm{C}-\mathrm{P}}=21.3 \mathrm{~Hz}, \mathrm{CH}=\right), 137.7\left(\mathrm{~d}, J_{\mathrm{C}-\mathrm{P}}=8.3 \mathrm{~Hz}, \mathrm{C}\right), 140.0\left(\mathrm{~d}, J_{\mathrm{C}-\mathrm{P}}=9.1 \mathrm{~Hz}, \mathrm{C}\right)$. TOF-MS (ESI+): $\mathrm{m} / \mathrm{z}=550.1546$, calcd. for $\mathrm{C}_{33} \mathrm{H}_{35} \mathrm{FePS}[\mathrm{M}]^{+}:$550.1547).

8-S: Yield $110 \mathrm{mg}(83 \%) .{ }^{31} \mathrm{P}\left\{{ }^{1} \mathrm{H}\right\}$ NMR (121 MHz, $\left.\mathrm{CDCl}_{3}\right), \delta$ : 41.4. ${ }^{1} \mathrm{H}$ NMR (300 $\mathrm{MHz}, \mathrm{CDCl}_{3}$ ), $\delta: 3.83(\mathrm{~m}, 1 \mathrm{H}, \mathrm{CH}=, \mathrm{Cp}), 4.26(\mathrm{~s}, 1 \mathrm{H}, \mathrm{CH}=, \mathrm{Cp}), 4.29\left(\mathrm{~d}, 1 \mathrm{H}, \mathrm{CH}_{2},{ }^{2} \mathrm{~J}_{\mathrm{H}-}\right.$ $\mathrm{H}=13.0 \mathrm{~Hz}), 4.32(\mathrm{~s}, 5 \mathrm{H}, \mathrm{CH}=, \mathrm{Cp}) 4.42(\mathrm{~s}, 1 \mathrm{H}, \mathrm{CH}=, \mathrm{Cp}), 4.60\left(\mathrm{~d}, 1 \mathrm{H}, \mathrm{CH}_{2},{ }^{2} \mathrm{~J}_{\mathrm{H}-\mathrm{H}}=\right.$ 13.0 Hz), 7.55-7.35 (m, 10H, CH=), 7.9-7.7 (m, 6H, CH=), $8.23(\mathrm{~m}, 1 \mathrm{H}, \mathrm{CH}=) .{ }^{13} \mathrm{C}\left\{{ }^{1} \mathrm{H}\right\}$ $\operatorname{NMR}\left(75 \mathrm{MHz}, \mathrm{CDCl}_{3}\right), \delta: 33.5\left(\mathrm{CH}_{2}\right), 69.2\left(\mathrm{~d}, J_{\mathrm{C}-\mathrm{P}}=10.4 \mathrm{~Hz}, \mathrm{CH}=, \mathrm{Cp},\right), 71.0(\mathrm{CH}=$, Cp), $73.8\left(\mathrm{~d}, J_{\mathrm{C}-\mathrm{P}}=9.2 \mathrm{~Hz}, \mathrm{CH}=, \mathrm{Cp},\right), 74.1\left(\mathrm{~d}, J_{\mathrm{C}-\mathrm{P}}=96.3 \mathrm{~Hz}, \mathrm{C}, \mathrm{Cp},\right), 74.6\left(\mathrm{~d}, J_{\mathrm{C}-\mathrm{P}}=\right.$ $12.7 \mathrm{~Hz}, \mathrm{CH}=, \mathrm{Cp},), 88.8\left(\mathrm{~d}, J_{\mathrm{C}-\mathrm{P}}=12.0 \mathrm{~Hz}, \mathrm{C}, \mathrm{Cp},\right), 125.4(\mathrm{CH}=), 125.5(\mathrm{CH}=)$, 126.1(CH=), $126.2(\mathrm{CH}=), 127.3(\mathrm{CH}=), 128.1\left(\mathrm{~d}, J_{\mathrm{C}-\mathrm{P}}=12.6 \mathrm{~Hz}, \mathrm{CH}=\right), 128.2\left(\mathrm{~d}, J_{\mathrm{C}-\mathrm{P}}=\right.$ $12.7 \mathrm{~Hz}, \mathrm{CH}=), 128.4(\mathrm{CH}=), 128.9(\mathrm{CH}=), 131.3\left(\mathrm{~d}, J_{\mathrm{C}-\mathrm{P}}=2.7 \mathrm{~Hz}, 2 \mathrm{C}\right), 132.1\left(\mathrm{~d}, J_{\mathrm{C}-\mathrm{P}}=\right.$ $10.8 \mathrm{~Hz}, \mathrm{CH}=), 132.2\left(\mathrm{~d}, J_{\mathrm{C}-\mathrm{P}}=10.7 \mathrm{~Hz}, \mathrm{CH}=\right), 132.9(\mathrm{C}), 133.6\left(\mathrm{~d}, J_{\mathrm{C}-\mathrm{P}}=70.2 \mathrm{~Hz}, \mathrm{C}\right)$, 133.9 (C), 134.0 (C), 134.5 (d, JC-P $=71 \mathrm{~Hz}, \mathrm{C}) . \mathrm{HR} / \mathrm{MS}$ (ESI) m/e: 574.0642 (M, 75 $\%$; calculated for $\mathrm{C}_{33} \mathrm{H}_{27} \mathrm{PS}_{2} \mathrm{Fe}$ : 574.0641), 415.0364(M-S(naphthyl), $100 \%$ ). 
8: Yield $92 \mathrm{mg}(89 \%) .{ }^{31} \mathrm{P}$ NMR (162 MHz, $\left.\mathrm{CDCl}_{3}\right), \delta$ : -24.1. ${ }^{1} \mathrm{H}$ NMR (400 MHz, $\left.\mathrm{CDCl}_{3}\right), \delta: 3.86(\mathrm{~m}, 1 \mathrm{H}, \mathrm{CH}=, \mathrm{Cp}), 4.04(\mathrm{~s}, 5 \mathrm{H}, \mathrm{CH}=, \mathrm{Cp}), 4.21\left(\mathrm{~b}, 2 \mathrm{H}, \mathrm{CH}_{2}\right), 4.29(\mathrm{~m}$, $1 \mathrm{H}, \mathrm{CH}=, \mathrm{Cp}), 4.43(\mathrm{~m}, 1 \mathrm{H}, \mathrm{CH}=, \mathrm{Cp}), 7.33$ (m, 5H, CH=), 7.4-7.5 (m, 4H, CH=), 7.53 (m, 3H, CH=), $7.61(\mathrm{~m}, 2 \mathrm{H}, \mathrm{CH}=), 7.76(\mathrm{~m}, 1 \mathrm{H}, \mathrm{CH}=), 7.85(\mathrm{~m}, 1 \mathrm{H}, \mathrm{CH}=), 8.30(\mathrm{~m}$, $1 \mathrm{H}, \mathrm{CH}=) .{ }^{13} \mathrm{C}\left\{{ }^{1} \mathrm{H}\right\} \mathrm{NMR}\left(100 \mathrm{MHz}, \mathrm{CDCl}_{3}\right), \delta: 34.6\left(\mathrm{~d}, J_{\mathrm{C}-\mathrm{P}}=12.2 \mathrm{~Hz}, \mathrm{CH}_{2}\right), 69.8$ $(\mathrm{CH}=, \mathrm{Cp}), 70.0(\mathrm{CH}=, \mathrm{Cp}), 71.6\left(\mathrm{~d}, J_{\mathrm{C}-\mathrm{P}}=3.8 \mathrm{~Hz}, \mathrm{CH}=, \mathrm{Cp},\right), 71.8\left(\mathrm{~d}, J_{\mathrm{C}-\mathrm{P}}=3.8 \mathrm{~Hz}\right.$, $\mathrm{CH}=, \mathrm{Cp},), 76.1\left(\mathrm{~d}, J_{\mathrm{C}-\mathrm{P}}=8.4 \mathrm{~Hz}, \mathrm{C}, \mathrm{Cp},\right), 89.9\left(\mathrm{~d}, J_{\mathrm{C}-\mathrm{P}}=25.9 \mathrm{~Hz}, \mathrm{C}, \mathrm{Cp},\right), 125.6\left(\mathrm{~d}, J_{\mathrm{C}-\mathrm{P}}\right.$ $=6.8 \mathrm{~Hz}, \mathrm{CH}=), 126.3\left(\mathrm{~d}, J_{\mathrm{C}-\mathrm{P}}=20.6 \mathrm{~Hz}, \mathrm{CH}=\right), 127.6(\mathrm{CH}=), 128.1(\mathrm{CH}=), 128.2(\mathrm{~d}$, $\left.J_{\mathrm{C}-\mathrm{P}}=6.1 \mathrm{~Hz}, \mathrm{CH}=\right), 128.4\left(\mathrm{~d}, J_{\mathrm{C}-\mathrm{P}}=5.9 \mathrm{~Hz}, \mathrm{CH}=\right), 128.6(\mathrm{CH}=), 129.3(\mathrm{CH}=), 132.6$ $(\mathrm{CH}=), 132.7(\mathrm{CH}=), 133.2(\mathrm{C}), 134.0(\mathrm{C}), 134.3(\mathrm{C}), 135.1(\mathrm{CH}=), 135.4(\mathrm{CH}=)$, $137.6\left(\mathrm{~d}, J_{\mathrm{C}-\mathrm{P}}=7.6 \mathrm{~Hz}, \mathrm{C}\right), 139.7\left(\mathrm{~d}, J_{\mathrm{C}-\mathrm{P}}=9.1 \mathrm{~Hz}, \mathrm{C}\right)$. TOF-MS (ESI+) $: \mathrm{m} / \mathrm{z}=$ 542.0919, calcd. for $\mathrm{C}_{33} \mathrm{H}_{27} \mathrm{FePS}[\mathrm{M}]^{+}$: 542.0921).

9-S: Yield $118 \mathrm{mg}(93 \%) .{ }^{31} \mathrm{P}\left\{{ }^{1} \mathrm{H}\right\} \mathrm{NMR}\left(162 \mathrm{MHz}, \mathrm{CDCl}_{3}\right), \delta: 41.3 .{ }^{1} \mathrm{H}$ NMR (400 $\left.\mathrm{MHz}, \mathrm{CDCl}_{3}\right), \delta: 2.42\left(\mathrm{~s}, 6 \mathrm{H}, \mathrm{CH}_{3}\right), 3.78(\mathrm{~m}, 1 \mathrm{H}, \mathrm{CH}=, \mathrm{Cp}), 3.86\left(\mathrm{~d}, 1 \mathrm{H}, \mathrm{CH}_{2},{ }^{2} \mathrm{~J}_{\mathrm{H}-\mathrm{H}}=\right.$ $12.7 \mathrm{~Hz}), 4.30(\mathrm{~m}, 1 \mathrm{H}, \mathrm{CH}=, \mathrm{Cp}), 4.33\left(\mathrm{~d}, 1 \mathrm{H}, \mathrm{CH}_{2},{ }^{2} J_{\mathrm{H}-\mathrm{H}}=12.7 \mathrm{~Hz}\right), 4.34(\mathrm{~s}, 5 \mathrm{H}, \mathrm{CH}=$, $\mathrm{Cp}), 4.45(\mathrm{~m}, 1 \mathrm{H}, \mathrm{CH}=, \mathrm{Cp}), 7.0-7.2(\mathrm{~m}, 3 \mathrm{H}, \mathrm{CH}=)$, 7.4-7.6 (m, 6H, CH=), 7.7-7.8 (m, $2 \mathrm{H}, \mathrm{CH}=), 7.8-7.9(\mathrm{~m}, 2 \mathrm{H}, \mathrm{CH}=) .{ }^{13} \mathrm{C}\left\{{ }^{1} \mathrm{H}\right\} \mathrm{NMR}\left(100 \mathrm{MHz}, \mathrm{CDCl}_{3}\right), \delta: 22.2\left(\mathrm{CH}_{3}\right)$, $33.6\left(\mathrm{CH}_{2}\right) 69.2\left(\mathrm{~d}, J_{\mathrm{C}-\mathrm{P}}=10.3 \mathrm{~Hz}, \mathrm{CH}=, \mathrm{Cp}\right), 70.9(\mathrm{CH}=, \mathrm{Cp}), 73.3\left(\mathrm{~d}, J_{\mathrm{C}-\mathrm{P}}=9.2 \mathrm{~Hz}\right.$, $\mathrm{CH}=, \mathrm{Cp}), 74.4\left(\mathrm{~d}, J_{\mathrm{C}-\mathrm{P}}=95.1 \mathrm{~Hz}, \mathrm{C}, \mathrm{Cp}\right), 74.5\left(\mathrm{~d}, J_{\mathrm{C}-\mathrm{P}}=12.5 \mathrm{~Hz}, \mathrm{CH}=, \mathrm{Cp}\right), 89.6\left(\mathrm{~d}, J_{\mathrm{C}-}\right.$ $\mathrm{P}=12.2 \mathrm{~Hz}, \mathrm{C}, \mathrm{Cp}), 128.0(\mathrm{CH}=), 128.1(\mathrm{CH}=), 128.1(\mathrm{CH}=), 128.2(\mathrm{CH}=), 131.2(\mathrm{~d}$, $\left.J_{\mathrm{C}-\mathrm{P}}=3.5 \mathrm{~Hz}, \mathrm{CH}=\right), 131.3\left(\mathrm{~d}, J_{\mathrm{C}-\mathrm{P}}=3.5 \mathrm{~Hz}, \mathrm{CH}=\right), 132.1\left(\mathrm{~d}, J_{\mathrm{C}-\mathrm{P}}=10.8 \mathrm{~Hz}, \mathrm{CH}=\right)$, $132.3\left(\mathrm{~d}, J_{\mathrm{C}-\mathrm{P}}=10.7 \mathrm{~Hz}, \mathrm{CH}=\right), 133.5\left(\mathrm{~d}, J_{\mathrm{C}-\mathrm{P}}=85.5 \mathrm{~Hz}, \mathrm{C}\right), 134.2(\mathrm{C}), 134.4\left(\mathrm{~d}, J_{\mathrm{C}-\mathrm{P}}=\right.$ $86.6 \mathrm{~Hz}, \mathrm{C}), 143.2(\mathrm{C})$.

9: Yield $101 \mathrm{mg}(91 \%) .{ }^{31} \mathrm{P} \mathrm{NMR}\left(162 \mathrm{MHz}, \mathrm{CDCl}_{3}\right), \delta:-24.0 .{ }^{1} \mathrm{H} \mathrm{NMR}(400 \mathrm{MHz}$, $\left.\mathrm{CDCl}_{3}\right), \delta: 2.43\left(\mathrm{~s}, 6 \mathrm{H}, \mathrm{CH}_{3}\right), 3.46\left(\mathrm{~d}, 1 \mathrm{H}, \mathrm{CH}_{2},{ }^{2} J_{\mathrm{H}-\mathrm{H}}=11.2 \mathrm{~Hz}\right), 3.79(\mathrm{~b}, 1 \mathrm{H}, \mathrm{CH}=$, $\mathrm{Cp}), 3.84\left(\mathrm{~d}, 1 \mathrm{H}, \mathrm{CH}_{2},{ }^{2} \mathrm{~J}_{\mathrm{H}-\mathrm{H}}=11.2 \mathrm{~Hz}\right), 4.00$ (s, 5H, CH=, Cp), 4.27 (b, 1H, CH=, Cp), 4.37 (b, 1H, CH=, Cp), 7.07 (m, 3H, $\mathrm{CH}=), 7.39$ (m, 5H, $\mathrm{CH}=), 7.55$ (m, 3H, CH=), $7.59(\mathrm{~m}, 2 \mathrm{H}, \mathrm{CH}=) .{ }^{13} \mathrm{C}\left\{{ }^{1} \mathrm{H}\right\} \mathrm{NMR}\left(100 \mathrm{MHz}, \mathrm{CDCl}_{3}\right), \delta: 22.0\left(\mathrm{CH}_{3}\right), 34.3\left(\mathrm{~d}, J_{\mathrm{C}-\mathrm{P}}=\right.$ $\left.12.9 \mathrm{~Hz}, \mathrm{CH}_{2}\right) 69.6(\mathrm{CH}=, \mathrm{Cp}), 69.7(\mathrm{CH}=, \mathrm{Cp}), 71.3(\mathrm{~b}, \mathrm{CH}=, \mathrm{Cp}), 75.9\left(\mathrm{~d}, J_{\mathrm{C}-\mathrm{P}}=7.6\right.$ 
$\mathrm{Hz}, \mathrm{C}, \mathrm{Cp}), 90.4$ (d, JC-P $=25.9 \mathrm{~Hz}, \mathrm{C}, \mathrm{Cp}), 127.8(\mathrm{CH}=), 127.9(\mathrm{CH}=), 128.0 \quad(\mathrm{CH}=)$, $128.1(\mathrm{CH}=), 128.2(\mathrm{CH}=), 129.1(\mathrm{CH}=), 132.5\left(\mathrm{~d}, J_{\mathrm{C}-\mathrm{P}}=18.2 \mathrm{~Hz}, \mathrm{CH}=\right), 134.2(\mathrm{C})$, $135.2\left(\mathrm{~d}, J_{\mathrm{C}-\mathrm{P}}=21.3 \mathrm{~Hz}, \mathrm{CH}=\right), 137.6\left(\mathrm{~d}, J_{\mathrm{C}-\mathrm{P}}=8.3 \mathrm{~Hz}, \mathrm{C}\right), 139.7\left(\mathrm{~d}, J_{\mathrm{C}-\mathrm{P}}=9.1 \mathrm{~Hz}, \mathrm{C}\right)$, 143.1 (C). TOF-MS (ESI+): $\mathrm{m} / \mathrm{z}=520.1074$, calcd. for $\mathrm{C}_{31} \mathrm{H}_{29} \mathrm{FePS}[\mathrm{M}]^{+}:$520.1077).

\section{General procedure for the preparation of $[\operatorname{Ir}(\operatorname{cod})(1-12)] B$ ArF $_{F}$}

The corresponding ligand $(0.074 \mathrm{mmol})$ was dissolved in $\mathrm{CH}_{2} \mathrm{Cl}_{2}(5 \mathrm{~mL})$ and $[\operatorname{Ir}(\mu-$ $\mathrm{Cl})(\mathrm{cod})]_{2}(25.0 \mathrm{mg}, 0.037 \mathrm{mmol})$ was added. The reaction mixture was refluxed at 40 ${ }^{\circ} \mathrm{C}$ for 1 hour. After $5 \mathrm{~min}$ at room temperature, $\mathrm{NaBAr}_{\mathrm{F}}(77.2 \mathrm{mg}, 0.080 \mathrm{mmol})$ and water $(5 \mathrm{~mL})$ were added and the reaction mixture was stirred vigorously for $30 \mathrm{~min}$ at room temperature. The phases were separated and the aqueous phase was extracted twice with $\mathrm{CH}_{2} \mathrm{Cl}_{2}$. The combined organic phases were dried with $\mathrm{MgSO}_{4}$. Evaporation of the solvent gave a brown-orange solid, which was purified by flash chromatography on neutral silica (dichloromethane/petroleum ether (1/1) as eluent) to produce the corresponding complex as an orange solid.

[Ir(cod)(1)]BArF: Yield $105.9 \mathrm{mg}(89 \%) .{ }^{31} \mathrm{P} \mathrm{NMR}\left(162 \mathrm{MHz}, \mathrm{CDCl}_{3}\right), \delta: 9.5$ (s); ${ }^{1} \mathrm{H}$ NMR $\left(400 \mathrm{MHz}, \mathrm{CDCl}_{3}\right), \delta: 1.12\left(\mathrm{t}, 3 \mathrm{H},{ }^{3} J_{\mathrm{H}-\mathrm{H}}=7.6 \mathrm{~Hz}, \mathrm{CH}_{3}\right), 1.56\left(\mathrm{~m}, 1 \mathrm{H}, \mathrm{CH}_{2}\right.$, cod), 1.71 (m, 1H, $\left.\mathrm{CH}_{2}, \mathrm{cod}\right), 2.06$ (m, 1H, $\left.\mathrm{CH}_{2}, \mathrm{cod}\right), 2.32$ (m, 1H, $\left.\mathrm{CH}_{2}, \mathrm{cod}\right), 2.45$ (m, $\left.3 \mathrm{H}, \mathrm{CH}_{2}, \mathrm{cod}\right), 2.64\left(\mathrm{~m}, 1 \mathrm{H}, \mathrm{CH}_{2}, \mathrm{Et}\right), 2.78\left(\mathrm{~d}, 1 \mathrm{H},{ }^{2} \mathrm{~J}_{\mathrm{H}-\mathrm{H}}=12.4 \mathrm{~Hz}, \mathrm{CH}_{2}-\mathrm{S}\right), 2.91(\mathrm{~m}$, $\left.1 \mathrm{H}, \mathrm{CH}_{2}, \mathrm{Et}\right), 3.47(\mathrm{~m}, 1 \mathrm{H}, \mathrm{CH}=, \mathrm{cod}), 3.57(\mathrm{~m}, 1 \mathrm{H}, \mathrm{CH}=, \operatorname{cod}), 4.00\left(\mathrm{~d}, 1 \mathrm{H},{ }^{2} J_{\mathrm{H}-\mathrm{H}}=\right.$ $12.4 \mathrm{~Hz}, \mathrm{CH}_{2}-\mathrm{S}$ ), 4.05 (s, 1H, $\left.\mathrm{CH}=, \mathrm{Cp}\right), 4.41$ (m, 1H, $\left.\mathrm{CH}=, \mathrm{Cp}\right), 4.47$ (s, 6H, CH=, $\mathrm{Cp}), 4.63(\mathrm{~m}, 1 \mathrm{H}, \mathrm{CH}=, \operatorname{cod}), 4.94(\mathrm{~m}, 1 \mathrm{H}, \mathrm{CH}=, \operatorname{cod}), 7.3-7.8(\mathrm{~m}, 22 \mathrm{H}, \mathrm{CH}=)$ ) ${ }^{13} \mathrm{C}$

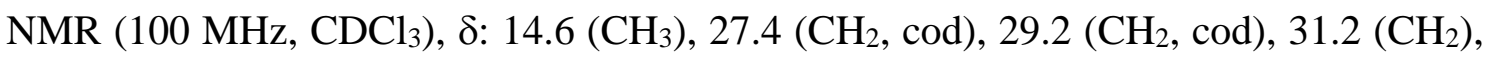
$31.6\left(\mathrm{CH}_{2}, \mathrm{cod}\right), 33.7\left(\mathrm{CH}_{2}, \mathrm{Et}\right), 35.0\left(\mathrm{CH}_{2}, \mathrm{cod}\right), 63.7$ (d, $\left.{ }^{1} J_{\mathrm{C}-\mathrm{P}}=63.6 \mathrm{~Hz}, \mathrm{C}, \mathrm{Cp}\right), 68.8$ $\left(\mathrm{d}, J_{\mathrm{C}-\mathrm{P}}=5.2 \mathrm{~Hz}, \mathrm{CH}=, \mathrm{Cp}\right), 70.9(\mathrm{CH}=, \operatorname{cod}$ and $\mathrm{CH}=, \mathrm{Cp}), 72.3(\mathrm{CH}=, \operatorname{cod}), 73.2$ $(\mathrm{CH}=, \mathrm{Cp}), 76.0\left(\mathrm{~d}, J_{\mathrm{C}-\mathrm{P}}=7.0 \mathrm{~Hz}_{\mathrm{s}} \mathrm{CH}=, \mathrm{Cp}\right), 84.3\left(\mathrm{~d},{ }^{2} J_{\mathrm{C}-\mathrm{P}}=15.5 \mathrm{~Hz}, \mathrm{C}, \mathrm{Cp}\right), 90.5(\mathrm{~d}$, $\left.J_{\mathrm{C}-\mathrm{P}}=14.6 \mathrm{~Hz}, \mathrm{CH}=, \mathrm{cod}\right), 90.7$ (d, $\left.J_{\mathrm{C}-\mathrm{P}}=14.6 \mathrm{~Hz}, \mathrm{CH}=. \operatorname{cod}\right), 117.4$ (b, CH=, BArF), 120.4-134.2 (aromatic carbons), 134.7 (b, $\mathrm{CH}=, \mathrm{BAr}_{\mathrm{F}}$ ), 161.7 (q, ${ }^{1} J_{\mathrm{C}-\mathrm{B}}=48.8 \mathrm{~Hz}, \mathrm{C}-\mathrm{B}$, BAr $\left._{F}\right)$. TOF-MS $(E S I+): \mathrm{m} / \mathrm{z}=745.1336$, calcd. for $\mathrm{C}_{65} \mathrm{H}_{49} \mathrm{BF}_{24}$ FeIrPS $\left[\mathrm{M}-\mathrm{BAr}_{\mathrm{F}}\right]^{+}$: 
745.1332). Anal. calcd (\%) for $\mathrm{C}_{65} \mathrm{H}_{49} \mathrm{BF}_{24} \mathrm{FeIrPS}$ : C, 48.55; H, 3.07; S, 1.99; found: $\mathrm{C}$, 48.34; H, 3.06; S, 1.95.

[Ir(cod)(2)]BArF: Yield $108.0 \mathrm{mg}(90 \%) .{ }^{31} \mathrm{P} \mathrm{NMR}\left(162 \mathrm{MHz}, \mathrm{CDCl}_{3}\right), \delta: 9.1$ (s); ${ }^{1} \mathrm{H}$ NMR (400 MHz, $\left.\mathrm{CDCl}_{3}\right), \delta: 1.18\left(\mathrm{~d},{ }^{3} \mathrm{~J}_{\mathrm{H}-\mathrm{H}}=6.8 \mathrm{~Hz}, 3 \mathrm{H}, \mathrm{CH}_{3},{ }^{i} \mathrm{Pr}\right), 1.41\left(\mathrm{~d}, 3 \mathrm{H},{ }^{3} \mathrm{~J}_{\mathrm{H}-\mathrm{H}}\right.$ $\left.=6.8 \mathrm{~Hz}, 3 \mathrm{H}, \mathrm{CH}_{3},{ }^{i} \mathrm{Pr}\right), 1.62\left(\mathrm{~m}, 1 \mathrm{H}, \mathrm{CH}_{2}, \mathrm{cod}\right), 1.78\left(\mathrm{~m}, 1 \mathrm{H}, \mathrm{CH}_{2}, \mathrm{cod}\right), 2.17(\mathrm{~m}, 2 \mathrm{H}$, $\mathrm{CH}_{2}$, cod), 2.41 (m, 1H, $\mathrm{CH}_{2}$, cod), 2.51 (m, 2H, $\left.\mathrm{CH}_{2}, \operatorname{cod}\right), 2.57$ (m, 1H, $\mathrm{CH}_{2}, \operatorname{cod}$ ), $2.84\left(\mathrm{~d}, 3 \mathrm{H},{ }^{2} J_{\mathrm{H}-\mathrm{H}}=11.6 \mathrm{~Hz}, \mathrm{CH}_{2}-\mathrm{S}\right), 3.24\left(\mathrm{q}, 1 \mathrm{H},{ }^{2} J_{\mathrm{H}-\mathrm{H}}=6.8 \mathrm{~Hz}, \mathrm{CH},{ }^{i} \mathrm{Pr}\right), 3.55(\mathrm{~m}, 1 \mathrm{H}$, $\mathrm{CH}=$, cod), $3.59(\mathrm{~m}, 1 \mathrm{H}, \mathrm{CH}=, \mathrm{Cp}), 4.05\left(\mathrm{~d}, 1 \mathrm{H}, J_{\mathrm{H}-\mathrm{H}}=11.6 \mathrm{~Hz}, \mathrm{CH}_{2}-\mathrm{S}\right), 4.08(\mathrm{~s}, 1 \mathrm{H}$, $\mathrm{CH}=, \mathrm{Cp}), 4.47$ (s, 1H, CH=, Cp), 4.54 (s, 5H, CH=, Cp), 4.57 (s, 1H, CH=, Cp), 4.81 $(\mathrm{m}, 1 \mathrm{H}, \mathrm{CH}=, \mathrm{cod}), 5.04(\mathrm{~m}, 1 \mathrm{H}, \mathrm{CH}=, \mathrm{cod}), 7.4-7.8(\mathrm{~m}, 22 \mathrm{H}, \mathrm{CH}=) ;{ }^{13} \mathrm{C} \mathrm{NMR}(100$ $\left.\mathrm{MHz}, \mathrm{CDCl}_{3}\right), \delta: 21.7\left(\mathrm{CH}_{3},{ }^{i} \mathrm{Pr}\right), 23.1\left(\mathrm{CH}_{3},{ }^{i} \mathrm{Pr}\right), 24.8\left(\mathrm{~b}, \mathrm{CH}_{2} \mathrm{~S}\right), 27.3\left(\mathrm{CH}_{2}, \mathrm{cod}\right), 29.0$ $\left(\mathrm{CH}_{2}, \mathrm{cod}\right), 31.5\left(\mathrm{CH}_{2}, \mathrm{cod}\right), 35.2\left(\mathrm{~b}, \mathrm{CH}_{2}, \mathrm{cod}\right), 43.3\left(\mathrm{CH},{ }^{i} \mathrm{Pr}\right), 63.7$ (d, ${ }^{1} J_{\mathrm{C}-\mathrm{P}}=63.9 \mathrm{~Hz}$, C, Cp), $68.7\left(\mathrm{~d}, J_{\mathrm{C}-\mathrm{P}}=6.2 \mathrm{~Hz}, \mathrm{CH}=, \mathrm{Cp}\right), 69.9(\mathrm{CH}=, \operatorname{cod}), 71.0(\mathrm{CH}=, \mathrm{Cp}), 71.5(\mathrm{CH}=$, $\operatorname{cod}), 73.4\left(\mathrm{~d},{ }^{3} J_{\mathrm{C}-\mathrm{P}}=2.1 \mathrm{~Hz}, \mathrm{CH}=, \mathrm{Cp}\right), 76.2\left(\mathrm{~d}, J_{\mathrm{C}-\mathrm{P}}=6.1 \mathrm{~Hz}, \mathrm{CH}=, \mathrm{Cp}\right), 84.0\left(\mathrm{~d},{ }^{2} J_{\mathrm{C}-\mathrm{P}}\right.$ $=16.0 \mathrm{~Hz}, \mathrm{C}, \mathrm{Cp}), 90.3\left(\mathrm{~d}, J_{\mathrm{C}-\mathrm{P}}=11.4 \mathrm{~Hz}, \mathrm{CH}=, \operatorname{cod}\right), 90.8\left(\mathrm{~d}, J_{\mathrm{C}-\mathrm{P}}=11.4 \mathrm{~Hz}, \mathrm{CH}=\right.$, cod), 117.4 (b, $\mathrm{CH}=, \mathrm{BAr}_{\mathrm{F}}$ ), 120.4-134.2 (aromatic carbons), 134.7 (b, $\mathrm{CH}=, \mathrm{BAr}_{\mathrm{F}}$ ), $161.7\left(\mathrm{q},{ }^{1} J_{\mathrm{C}-\mathrm{B}}=50.1 \mathrm{~Hz}, \mathrm{C}-\mathrm{B}, \mathrm{BAr}\right)$. TOF-MS (ESI+): $\mathrm{m} / \mathrm{z}=759.1485$, calcd. for $\mathrm{C}_{66} \mathrm{H}_{51} \mathrm{BF}_{24} \mathrm{FeIrPS}[\mathrm{M}-\mathrm{BAr}]^{+}$: 759.1489. Anal. calcd (\%) for $\mathrm{C}_{66} \mathrm{H}_{51} \mathrm{BF}_{24} \mathrm{FeIrPS}: \mathrm{C}$, 48.87; H, 3.17; S, 1.98; found: C, 48.69; H, 3.15; S, 1.95 .

[Ir(cod)(3)]BArF: Yield $111.9 \mathrm{mg}(91 \%) .{ }^{31} \mathrm{P} \mathrm{NMR}\left(162 \mathrm{MHz}, \mathrm{CDCl}_{3}\right), \delta: 9.1$ (s); ${ }^{1} \mathrm{H}$ NMR (400 MHz, $\left.\mathrm{CDCl}_{3}\right), \delta: 1.14\left(\mathrm{~m}, 2 \mathrm{H}, \mathrm{CH}_{2}\right), 1.51\left(\mathrm{~m}, 4 \mathrm{H}, \mathrm{CH}_{2}\right), 1.76(\mathrm{~m}, 1 \mathrm{H}$, $\mathrm{CH}_{2}$ ), 1.91 (m, 2H, $\left.\mathrm{CH}_{2}\right), 2.09\left(\mathrm{~m}, 1 \mathrm{H}, \mathrm{CH}_{2}\right), 2.17\left(\mathrm{~m}, 2 \mathrm{H}, \mathrm{CH}_{2}\right), 2.43\left(\mathrm{~m}, 1 \mathrm{H}, \mathrm{CH}_{2}\right)$, $2.51\left(\mathrm{~m}, 2 \mathrm{H}, \mathrm{CH}_{2}\right), 2.57\left(\mathrm{~m}, 1 \mathrm{H}, \mathrm{CH}_{2}\right), 2.85(\mathrm{~m}, 1 \mathrm{H}, \mathrm{CH}-\mathrm{S}), 2.90\left(\mathrm{~d}, 1 \mathrm{H},{ }^{2} J_{\mathrm{H}-\mathrm{H}}=10.8\right.$ $\left.\mathrm{Hz}, \mathrm{CH}_{2}-\mathrm{S}\right), 3.51(\mathrm{~m}, 1 \mathrm{H}, \mathrm{CH}=, \mathrm{cod}), 3.54(\mathrm{~m}, 1 \mathrm{H}, \mathrm{CH}=, \operatorname{cod}), 4.06\left(\mathrm{~d}, 1 \mathrm{H},{ }^{2} J_{\mathrm{H}-\mathrm{H}}=10.8\right.$ $\mathrm{Hz}, \mathrm{CH}_{2}-\mathrm{S}$ ), 4.08 (s, 1H, CH=, Cp), 4.46 (b, 1H, CH=, Cp), 4.53 (s, 6H, CH=, Cp), 4.76 $(\mathrm{m}, 1 \mathrm{H}, \mathrm{CH}=, \mathrm{cod}), 5.04(\mathrm{~m}, 1 \mathrm{H}, \mathrm{CH}=, \mathrm{cod}), 7.4-7.8(\mathrm{~m}, 22 \mathrm{H}, \mathrm{CH}=) ;{ }^{13} \mathrm{C} \mathrm{NMR}(100$ $\left.\mathrm{MHz}, \mathrm{CDCl}_{3}\right), \delta: 24.4\left(\mathrm{CH}_{2}\right), 26.0\left(\mathrm{~d},{ }^{3} \mathrm{~J}_{\mathrm{C}-\mathrm{P}}=3.8 \mathrm{~Hz}, \mathrm{CH}_{2}-\mathrm{S}\right), 27.1\left(\mathrm{CH}_{2}\right), 27.3\left(\mathrm{CH}_{2}\right)$, $28.7\left(\mathrm{CH}_{2}\right), 29.7\left(\mathrm{CH}_{2}\right), 31.5\left(\mathrm{CH}_{2}\right), 32.3\left(\mathrm{CH}_{2}\right), 34.1\left(\mathrm{CH}_{2}\right), 35.2\left(\mathrm{~d},{ }^{3} J_{\mathrm{C}-\mathrm{P}}=3.8 \mathrm{~Hz}\right.$, $\left.\mathrm{CH}_{2}-\mathrm{S}\right), 51.9(\mathrm{CH}-\mathrm{S}), 64.2\left(\mathrm{~d},{ }^{1} J_{\mathrm{C}-\mathrm{P}}=63.8 \mathrm{~Hz}, \mathrm{C}, \mathrm{Cp}\right), 68.6\left(\mathrm{~d}, J_{\mathrm{C}-\mathrm{P}}=6.1 \mathrm{~Hz}, \mathrm{CH}=, \mathrm{Cp}\right)$, 
$69.6(\mathrm{CH}=, \operatorname{cod}), 71.0(\mathrm{CH}=, \mathrm{Cp}), 71.3(\mathrm{CH}=, \operatorname{cod}), 73.3\left(\mathrm{~d}, J_{\mathrm{C}-\mathrm{P}}=3.1 \mathrm{~Hz}, \mathrm{CH}=, \mathrm{Cp}\right)$, $76.1\left(\mathrm{~d}, J_{\mathrm{C}-\mathrm{P}}=6.9 \mathrm{~Hz}, \mathrm{CH}=, \mathrm{Cp}\right), 84.4\left(\mathrm{~d},{ }^{2} J_{\mathrm{C}-\mathrm{P}}=16.7 \mathrm{~Hz}, \mathrm{C}, \mathrm{Cp}\right), 90.2\left(\mathrm{~d}, J_{\mathrm{C}-\mathrm{P}}=11.4\right.$ $\mathrm{Hz}, \mathrm{CH}=, \operatorname{cod}), 90.7\left(\mathrm{~d}, J_{\mathrm{C}-\mathrm{P}}=11.4 \mathrm{~Hz}, \mathrm{CH}=, \operatorname{cod}\right), 117.4\left(\mathrm{~b}, \mathrm{CH}=, \mathrm{BAr}_{\mathrm{F}}\right), 120.4-134.3$ (aromatic carbons), 134.7 (b, CH=, BAr ${ }_{\mathrm{F}}$ ), 161.7 (q, ${ }^{1} J_{\mathrm{C}-\mathrm{B}}=50.1 \mathrm{~Hz}, \mathrm{C}-\mathrm{B}, \mathrm{BAr}$ ). TOFMS (ESI+): $\mathrm{m} / \mathrm{z}=799.1779$, calcd. for $\mathrm{C}_{69} \mathrm{H}_{55} \mathrm{BF}_{24} \mathrm{FeIrPS}\left[\mathrm{M}-\mathrm{BAr}_{\mathrm{F}}\right]^{+}:$799.1802. Anal. calcd (\%) for $\mathrm{C}_{69} \mathrm{H}_{55} \mathrm{BF}_{24} \mathrm{FeIrPS}$ : C, 49.86; H, 3.34; S, 1.93; found: C, 49.76; H, 3.31; S, 1.90 .

[Ir(cod)(4)]BArF: Yield 108.9 mg (90\%). ${ }^{31} \mathrm{P}$ NMR (162 MHz, $\left.\mathrm{CDCl}_{3}\right), \delta: 10.3$ (s); ${ }^{1} \mathrm{H}$ NMR (400 MHz, $\mathrm{CDCl}_{3}$ ), $\delta: 1.35\left(\mathrm{~s}, 9 \mathrm{H}, \mathrm{CH}_{3},{ }^{t} \mathrm{Bu}\right), 1.52\left(\mathrm{~m}, 1 \mathrm{H}, \mathrm{CH}_{2}, \mathrm{cod}\right), 1.69$ (m, 1H, $\left.\mathrm{CH}_{2}, \operatorname{cod}\right), 2.18$ (m, 2H, $\mathrm{CH}_{2}$, cod), 2.37 (m, 1H, $\left.\mathrm{CH}_{2}, \operatorname{cod}\right), 2.53$ (m, 3H, $\mathrm{CH}_{2}$, $\operatorname{cod}), 2.80\left(\mathrm{~d}, 1 \mathrm{H},{ }^{2} J_{\mathrm{H}-\mathrm{H}}=12.0 \mathrm{~Hz}, \mathrm{CH}_{2}-\mathrm{S}\right), 3.51(\mathrm{~m}, 2 \mathrm{H}, \mathrm{CH}=, \operatorname{cod}), 4.15\left(\mathrm{~d}, 1 \mathrm{H},{ }^{2} J_{\mathrm{H}-\mathrm{H}}=\right.$ $12.0 \mathrm{~Hz}, \mathrm{CH}_{2}-\mathrm{S}$ ), 4.17 (s, 1H, CH=, Cp), 4.48 (s, 1H, CH=, Cp), 4.56 (m, 6H, CH=, $\mathrm{Cp}), 5.35\left(\mathrm{~m}, 1 \mathrm{H}, \mathrm{CH}=\right.$, cod), $5.45(\mathrm{~m}, 1 \mathrm{H}, \mathrm{CH}=$, cod), 7.4-7.8 (m, 22H, $\mathrm{CH}=)$; ${ }^{13} \mathrm{C}$ NMR (100 MHz, $\left.\mathrm{CDCl}_{3}\right), \delta: 27.4\left(\mathrm{CH}_{2}, \mathrm{cod}\right), 28.4$ (d, JC-P $\left.=2.4 \mathrm{~Hz}, \mathrm{CH}_{2}, \mathrm{cod}\right), 30.3$ (d, $\left.{ }^{3} J_{\mathrm{C}-\mathrm{P}}=4.6 \mathrm{~Hz}, \mathrm{CH}_{2}-\mathrm{S}\right), 31.8\left(\mathrm{CH}_{3},{ }^{t} \mathrm{Bu}\right), 32.1\left(\mathrm{~d}, J_{\mathrm{C}-\mathrm{P}}=2.3 \mathrm{~Hz}, \mathrm{CH}_{2}, \operatorname{cod}\right), 35.7\left(\mathrm{~d}, J_{\mathrm{C}-\mathrm{P}}=\right.$ $\left.4.7 \mathrm{~Hz}, \mathrm{CH}_{2}, \mathrm{cod}\right), 59.1\left(\mathrm{C},{ }^{t} \mathrm{Bu}\right), 62.6\left(\mathrm{~d},{ }^{1} J_{\mathrm{C}-\mathrm{P}}=60.4 \mathrm{~Hz}, \mathrm{C}, \mathrm{Cp}\right), 68.2(\mathrm{CH}=, \operatorname{cod}), 69.0$ $\left(\mathrm{d}, J_{\mathrm{C}-\mathrm{P}}=6.2 \mathrm{~Hz}, \mathrm{CH}=, \mathrm{Cp}\right), 69.1(\mathrm{CH}=, \operatorname{cod}), 71.1(\mathrm{CH}=, \mathrm{Cp}), 73.9\left(\mathrm{~d}, J_{\mathrm{C}-\mathrm{P}}=3.1 \mathrm{~Hz}\right.$, $\mathrm{CH}=, \mathrm{Cp}), 76.5\left(\mathrm{~d}, J_{\mathrm{C}-\mathrm{P}}=7.0 \mathrm{~Hz}, \mathrm{CH}=, \mathrm{Cp}\right), 84.8\left(\mathrm{~d},{ }^{2} J_{\mathrm{C}-\mathrm{P}}=16.3 \mathrm{~Hz}, \mathrm{C}, \mathrm{Cp}\right), 89.8$ (d, $\left.J_{\mathrm{C}-\mathrm{P}}=10.9 \mathrm{~Hz}, \mathrm{CH}=, \operatorname{cod}\right), 90.5\left(\mathrm{~d}, J_{\mathrm{C}-\mathrm{P}}=11.6 \mathrm{~Hz}, \mathrm{CH}=, \operatorname{cod}\right), 117.6\left(\mathrm{~b}, \mathrm{CH}=, \mathrm{BAr}_{\mathrm{F}}\right)$, 120.6-134.3 (aromatic carbons), 134.9 (b, CH=, BAr $), 161.8$ (q, ${ }^{1} J_{\mathrm{C}-\mathrm{B}}=50.4 \mathrm{~Hz}, \mathrm{C}-\mathrm{B}$, BAr $\left.{ }_{F}\right)$ TOF-MS $(E S I+): \mathrm{m} / \mathrm{z}=773.1644$, calcd. for $\mathrm{C}_{67} \mathrm{H}_{53} \mathrm{BF}_{24}$ FeIrPS $\left[\mathrm{M}-\mathrm{BAr}_{\mathrm{F}}\right]^{+}$: 773.1645. Anal. calcd (\%) for $\mathrm{C}_{67} \mathrm{H}_{53} \mathrm{BF}_{24}$ FeIrPS: C, 49.19; H, 3.27; S, 1.96; found: $\mathrm{C}$, 49.11; H, 3.25; S, 1.95. Suitable crystals for X-ray diffraction were achieved by slow diffusion of petroleum ether to an isopropanol solution.

[Ir(cod)(5)]BArF: Yield $114.2 \mathrm{mg}(90 \%) .{ }^{31} \mathrm{P}$ NMR (162 MHz, $\left.\mathrm{CDCl}_{3}\right), \delta: 10.3$ (s); ${ }^{1} \mathrm{H}$ NMR (400 MHz, $\left.\mathrm{CDCl}_{3}\right), \delta: 1.28(\mathrm{~m}, 1 \mathrm{H}, \mathrm{CH}), 1.48$ (m, 1H, $\left.\mathrm{CH}_{2}, \mathrm{cod}\right), 1.59-1.72$ (m, 6H, $\mathrm{CH}_{2}$ ), 1.84 (m, 4H, $\mathrm{CH}_{2}$ ), 2.05-2.17 (m, 6H, $\mathrm{CH}_{2}$ ), 2.19 (m, 3H, $\left.\mathrm{CH}_{2}, \mathrm{cod}\right), 2.33$ (m, 1H, $\left.\mathrm{CH}_{2}, \operatorname{cod}\right), 2.53\left(\mathrm{~m}, 2 \mathrm{H}, \mathrm{CH}_{2}, \operatorname{cod}\right), 2.58\left(\mathrm{~m}, 1 \mathrm{H}, \mathrm{CH}_{2}, \operatorname{cod}\right), 2.82\left(\mathrm{~d}, 1 \mathrm{H},{ }^{2} J_{\mathrm{H}-\mathrm{H}}=\right.$ $\left.12.4 \mathrm{~Hz}, \mathrm{CH}_{2}-\mathrm{S}\right), 4.17$ (s, $\left.1 \mathrm{H}, \mathrm{CH}=, \mathrm{Cp}\right), 3.48$ (m, 2H, CH=, cod), $4.13\left(\mathrm{~d}, 1 \mathrm{H},{ }^{2} J_{\mathrm{H}-\mathrm{H}}=\right.$ 
$\left.12.4 \mathrm{~Hz}, \mathrm{CH}_{2}-\mathrm{S}\right), 4.18$ (b, 1H, $\left.\mathrm{CH}=, \mathrm{Cp}\right), 4.46$ (b, 1H, $\left.\mathrm{CH}=, \mathrm{Cp}\right), 4.51$ (b, 1H, $\left.\mathrm{CH}=, \mathrm{Cp}\right)$, 4.55 (s, 5H, CH=, Cp), 5.49 (m, 2H, CH=, cod), 7.4-7.8 (m, 22H, CH=); ${ }^{13} \mathrm{C}$ NMR (100 $\left.\mathrm{MHz}, \mathrm{CDCl}_{3}\right), \delta: 22.6\left(\mathrm{CH}_{2}, \mathrm{Ad}\right), 27.2\left(\mathrm{CH}_{2}, \mathrm{cod}\right), 27.8\left(\mathrm{~d}, J_{\mathrm{C}-\mathrm{P}}=4.6 \mathrm{~Hz}, \mathrm{CH}_{2}, \mathrm{cod}\right)$, $27.9\left(\mathrm{CH}_{2}-\mathrm{S}\right), 30.5\left(\mathrm{CH}_{2}, \mathrm{Ad}\right), 32.0\left(\mathrm{CH}_{2}, \mathrm{cod}\right), 35.3\left(\mathrm{CH}_{2}, \mathrm{Ad}\right), 35.6\left(\mathrm{~d}, J_{\mathrm{C}-\mathrm{P}}=4.5 \mathrm{~Hz}\right.$, $\left.\mathrm{CH}_{2}, \operatorname{cod}\right), 43.9\left(\mathrm{CH}_{2}, \mathrm{Ad}\right), 62.7\left(\mathrm{~d},{ }^{1} J_{\mathrm{C}-\mathrm{P}}=63.8 \mathrm{~Hz}, \mathrm{C}, \mathrm{Cp}\right), 67.4(\mathrm{CH}=, \operatorname{cod}), 68.6$ $(\mathrm{CH}=, \mathrm{cod}), 68.7\left(\mathrm{~d}, J_{\mathrm{C}-\mathrm{P}}=6.0 \mathrm{~Hz}, \mathrm{CH}=, \mathrm{Cp}\right), 70.9(\mathrm{CH}=, \mathrm{Cp}), 73.6\left(\mathrm{~d}, J_{\mathrm{C}-\mathrm{P}}=3.8 \mathrm{~Hz}\right.$, $\mathrm{CH}=, \mathrm{Cp}), 76.2\left(\mathrm{~d}, J_{\mathrm{C}-\mathrm{P}}=7.6 \mathrm{~Hz}, \mathrm{CH}=, \mathrm{Cp}\right), 84.7$ (d, $\left.{ }^{2} J_{\mathrm{C}-\mathrm{P}}=16.7 \mathrm{~Hz}, \mathrm{C}, \mathrm{Cp}\right), 89.7$ (d, $\left.J_{\mathrm{C}-\mathrm{P}}=10.6 \mathrm{~Hz}, \mathrm{CH}=, \operatorname{cod}\right), 90.5$ (d, $\left.J_{\mathrm{C}-\mathrm{P}}=12.2 \mathrm{~Hz}, \mathrm{CH}=, \operatorname{cod}\right), 117.4\left(\mathrm{~b}, \mathrm{CH}=, \mathrm{BAr}_{\mathrm{F}}\right)$, 120.4-134.4 (aromatic carbons), 134.7 (b, $\mathrm{CH}=, \mathrm{BAr}_{\mathrm{F}}$ ), 161.7 (q, ${ }^{1} J_{\mathrm{C}-\mathrm{B}}=50.2 \mathrm{~Hz}, \mathrm{C}-\mathrm{B}$, $\left.\mathrm{BAr}_{\mathrm{F}}\right)$. TOF-MS $(\mathrm{ESI}+): \mathrm{m} / \mathrm{z}=851.2112$, calcd. for $\mathrm{C}_{73} \mathrm{H}_{59} \mathrm{BF}_{24} \mathrm{FeIrPS}\left[\mathrm{M}-\mathrm{BAr}_{\mathrm{F}}\right]^{+}$: 851.2115. Anal. calcd (\%) for $\mathrm{C}_{73} \mathrm{H}_{59} \mathrm{BF}_{24}$ FeIrPS: C, 51.15; H, 3.47; S, 1.87; found: C, 51.11; H, 3.44; S, 1.85.

[Ir(cod)(6)]BArF: Yield $109.1 \mathrm{mg}(89 \%) .{ }^{31} \mathrm{P} \mathrm{NMR}\left(162 \mathrm{MHz}, \mathrm{CDCl}_{3}\right), \delta: 8.8$ (s); ${ }^{1} \mathrm{H}$ NMR (400 MHz, $\mathrm{CDCl}_{3}$ ), $\delta: 1.72$ (m, 2H, $\left.\mathrm{CH}_{2}, \mathrm{cod}\right), 1.96$ (m, 1H, $\left.\mathrm{CH}_{2}-\mathrm{S}, \operatorname{cod}\right), 2.24$ (m, 1H, $\left.\mathrm{CH}_{2}, \operatorname{cod}\right), 2.46$ (m, 2H, $\left.\mathrm{CH}_{2}, \operatorname{cod}\right), 2.57$ (m, 2H, $\left.\mathrm{CH}_{2}, \operatorname{cod}\right), 3.31\left(\mathrm{~d}, 1 \mathrm{H},{ }^{2} J_{\mathrm{H}-\mathrm{H}}=\right.$ $\left.12.8 \mathrm{~Hz}, \mathrm{CH}_{2}\right), 3.70(\mathrm{~m}, 1 \mathrm{H}, \mathrm{CH}=, \mathrm{cod}), 3.72(\mathrm{~m}, 1 \mathrm{H}, \mathrm{CH}=, \operatorname{cod}), 3.94(\mathrm{~m}, 1 \mathrm{H}, \mathrm{CH}=$, cod), $4.17(\mathrm{~s}, 1 \mathrm{H}, \mathrm{CH}=, \mathrm{Cp}), 4.43\left(\mathrm{~d}, 1 \mathrm{H},{ }^{2} J_{\mathrm{H}-\mathrm{H}}=12.8 \mathrm{~Hz}, \mathrm{CH}_{2}\right), 4.51(\mathrm{~s}, 1 \mathrm{H}, \mathrm{CH}=, \mathrm{Cp})$, 4.59 (s, 1H, CH=, Cp), 4.65 (s, 5H, CH=, Cp), 4.88 (m, 1H, CH=, cod), 7.3-7.8 (b, 27H, $\mathrm{CH}=) ;{ }^{13} \mathrm{C} \mathrm{NMR}\left(100 \mathrm{MHz}, \mathrm{CDCl}_{3}\right), \delta: 27.1\left(\mathrm{CH}_{2}, \operatorname{cod}\right), 29.8\left(\mathrm{~d}, J_{\mathrm{C}-\mathrm{P}}=2.4 \mathrm{~Hz}, \mathrm{CH}_{2}\right.$, $\operatorname{cod}), 30.9\left(\mathrm{~d}, J_{\mathrm{C}-\mathrm{P}}=2.4 \mathrm{~Hz}, \mathrm{CH}_{2}, \operatorname{cod}\right), 34.8\left(\mathrm{~d}, J_{\mathrm{C}-\mathrm{P}}=4.6 \mathrm{~Hz}, \mathrm{CH}_{2}, \operatorname{cod}\right), 38.7\left(\mathrm{~d},{ }^{3} J_{\mathrm{C}-\mathrm{P}}=\right.$ $\left.3.5 \mathrm{~Hz}, \mathrm{CH}_{2}-\mathrm{S}\right), 64.2\left(\mathrm{~d},{ }^{1} J_{\mathrm{C}-\mathrm{P}}=63.6 \mathrm{~Hz}, \mathrm{C}, \mathrm{Cp}\right), 68.6\left(\mathrm{~d}, J_{\mathrm{C}-\mathrm{P}}=7.0 \mathrm{~Hz}, \mathrm{CH}=, \mathrm{Cp}\right), 70.9$ $(\mathrm{CH}=, \operatorname{cod}), 71.1(\mathrm{CH}=, \mathrm{Cp}), 72.9(\mathrm{CH}=, \operatorname{cod}), 73.5\left(\mathrm{~d}, J_{\mathrm{C}-\mathrm{P}}=3.2 \mathrm{~Hz}, \mathrm{CH}=, \mathrm{Cp}\right), 76.2$ $\left(\mathrm{d}, J_{\mathrm{C}-\mathrm{P}}=6.2 \mathrm{~Hz}, \mathrm{CH}=, \mathrm{Cp}\right), 84.0\left(\mathrm{~d},{ }^{2} J_{\mathrm{C}-\mathrm{P}}=16.3 \mathrm{~Hz}, \mathrm{C}, \mathrm{Cp}\right), 90.8\left(\mathrm{~d}, J_{\mathrm{C}-\mathrm{P}}=11.7 \mathrm{~Hz}\right.$, $\mathrm{CH}=, \operatorname{cod}), 94.4\left(\mathrm{~d}, J_{\mathrm{C}-\mathrm{P}}=10.8 \mathrm{~Hz}, \mathrm{CH}=, \operatorname{cod}\right), 117.4\left(\mathrm{~b}, \mathrm{CH}=, \mathrm{BAr}_{\mathrm{F}}\right), 120.4-134.2$ (aromatic carbons), 134.7 (b, CH=, BAr ${ }_{\mathrm{F}}$ ), 161.6 (q, ${ }^{1} J_{\mathrm{C}-\mathrm{B}}=49.6 \mathrm{~Hz}, \mathrm{C}-\mathrm{B}, \mathrm{BAr}_{\mathrm{F}}$ ). TOFMS (ESI+): $\mathrm{m} / \mathrm{z}=793.1330$, calcd. for $\mathrm{C}_{69} \mathrm{H}_{49} \mathrm{BF}_{24} \mathrm{Fe} \operatorname{IrPS}\left[\mathrm{M}-\mathrm{BAr}_{\mathrm{F}}\right]^{+}:$793.1332. Anal. calcd (\%) for $\mathrm{C}_{69} \mathrm{H}_{49} \mathrm{BF}_{24} \mathrm{Fe} I r P S: \mathrm{C}, 50.04 ; \mathrm{H}, 2.98 ; \mathrm{S}, 1.94$; found: C, 49.98; H, 2.96; S, 1.92. 
[Ir(cod)(7)]BArF: Yield $112.5 \mathrm{mg}(91 \%) .{ }^{31} \mathrm{P} \mathrm{NMR}\left(162 \mathrm{MHz}, \mathrm{CDCl}_{3}\right), \delta: 8.9$ (s); ${ }^{1} \mathrm{H}$ NMR (400 MHz, $\mathrm{CDCl}_{3}$ ), $\delta: 1.71$ (m, 2H, $\left.\mathrm{CH}_{2}, \mathrm{cod}\right), 1.85$ (m, 1H, $\left.\mathrm{CH}_{2}, \mathrm{cod}\right), 2.21$ (m, 2H, $\left.\mathrm{CH}_{2}, \mathrm{cod}\right), 2.41\left(\mathrm{~m}, 1 \mathrm{H}, \mathrm{CH}_{2}, \mathrm{cod}\right), 2.54$ (m, 3H, $\left.\mathrm{CH}_{2}, \operatorname{cod}\right), 2.67\left(\mathrm{~d}, 1 \mathrm{H},{ }^{2} \mathrm{~J}_{\mathrm{H}-\mathrm{H}}=\right.$ $\left.12.4 \mathrm{~Hz}, \mathrm{CH}_{2}\right), 3.64(\mathrm{~m}, 1 \mathrm{H}, \mathrm{CH}=, \operatorname{cod}), 3.72(\mathrm{~m}, 1 \mathrm{H}, \mathrm{CH}=, \operatorname{cod}), 3.76\left(\mathrm{~d}, 1 \mathrm{H},{ }^{2} J_{\mathrm{H}-\mathrm{H}}=\right.$ $\left.12.4 \mathrm{~Hz}, \mathrm{CH}_{2}\right), 3.83\left(\mathrm{~d}, 1 \mathrm{H},{ }^{2} \mathrm{~J}_{\mathrm{H}-\mathrm{H}}=13.2 \mathrm{~Hz}, \mathrm{CH}_{2}-\mathrm{Ph}\right), 4.11(\mathrm{~s}, 1 \mathrm{H}, \mathrm{CH}=, \mathrm{Cp}), 4.22$ (d, $\left.1 \mathrm{H},{ }^{2} J_{\mathrm{H}-\mathrm{H}}=13.2 \mathrm{~Hz}, \mathrm{CH}_{2}-\mathrm{Ph}\right), 4.41(\mathrm{~s}, 1 \mathrm{H}, \mathrm{CH}=, \mathrm{Cp}), 4.42(\mathrm{~s}, 1 \mathrm{H}, \mathrm{CH}=, \mathrm{Cp}), 4.47$ (s, $5 \mathrm{H}, \mathrm{CH}=, \mathrm{Cp}), 4.90(\mathrm{~m}, 1 \mathrm{H}, \mathrm{CH}=, \mathrm{cod}), 5.13(\mathrm{~m}, 1 \mathrm{H}, \mathrm{CH}=, \operatorname{cod}), 7.1-7.8(\mathrm{~b}, 27 \mathrm{H}$, $\mathrm{CH}=) ;{ }^{13} \mathrm{C}$ NMR $\left(100 \mathrm{MHz}, \mathrm{CDCl}_{3}\right), \delta: 27.9\left(\mathrm{~d}, J_{\mathrm{C}-\mathrm{P}}=1.6 \mathrm{~Hz}, \mathrm{CH}_{2}, \operatorname{cod}\right), 29.6\left(\mathrm{~d}, J_{\mathrm{C}-\mathrm{P}}=\right.$ $\left.2.4 \mathrm{~Hz}, \mathrm{CH}_{2}, \operatorname{cod}\right), 31.8$ (d, $J_{\mathrm{C}-\mathrm{P}}=2.3 \mathrm{~Hz}, \mathrm{CH}_{2}, \operatorname{cod}$ ), 32.8 (d, $J_{\mathrm{C}-\mathrm{P}}=4.7 \mathrm{~Hz}, \mathrm{CH}_{2}-\mathrm{S}$ ), $35.1\left(\mathrm{~d}, J_{\mathrm{C}-\mathrm{P}}=4.7 \mathrm{~Hz}, \mathrm{CH}_{2}, \mathrm{cod}\right), 44.5\left(\mathrm{CH}_{2}-\mathrm{Ph}\right), 64.5\left(\mathrm{~d},{ }^{1} J_{\mathrm{C}-\mathrm{P}}=63.6 \mathrm{~Hz}, \mathrm{C}, \mathrm{Cp}\right), 69.1$ $\left(\mathrm{d}, J_{\mathrm{C}-\mathrm{P}}=7.0 \mathrm{~Hz}, \mathrm{CH}=, \mathrm{Cp}\right), 71.2(\mathrm{CH}=, \mathrm{Cp}), 72.1(\mathrm{CH}=, \operatorname{cod}), 73.4(\mathrm{CH}=, \operatorname{cod}), 73.5$ $\left(\mathrm{d}, J_{\mathrm{C}-\mathrm{P}}=3.9 \mathrm{~Hz}, \mathrm{CH}=, \mathrm{Cp}\right), 76.2\left(\mathrm{~d}, J_{\mathrm{C}-\mathrm{P}}=7.0 \mathrm{~Hz}, \mathrm{CH}=, \mathrm{Cp}\right), 84.3\left(\mathrm{~d},{ }^{2} J_{\mathrm{C}-\mathrm{P}}=16.3 \mathrm{~Hz}\right.$, C, Cp), 90.7 (d, $\left.J_{\mathrm{C}-\mathrm{P}}=11.6 \mathrm{~Hz}, \mathrm{CH}=, \operatorname{cod}\right), 91.2\left(\mathrm{~d}, J_{\mathrm{C}-\mathrm{P}}=11.6 \mathrm{~Hz}, \mathrm{CH}=, \operatorname{cod}\right), 117.6$ (b, $\mathrm{CH}=, \mathrm{BAr}_{\mathrm{F}}$ ), 120.6-134.2 (aromatic carbons), $135.0\left(\mathrm{~b}, \mathrm{CH}=, \mathrm{BAr}_{\mathrm{F}}\right), 161.8$ (q, ${ }^{1} J_{\mathrm{C}-\mathrm{B}}=$ $49.7 \mathrm{~Hz}, \mathrm{C}-\mathrm{B}, \mathrm{BAr}$ ) . TOF-MS (ESI+): $\mathrm{m} / \mathrm{z}=807.1488$, calcd. for $\mathrm{C}_{70} \mathrm{H}_{51} \mathrm{BF}_{24} \mathrm{FeIrPS}$ $\left[\mathrm{M}-\mathrm{BAr}_{\mathrm{F}}\right]^{+}$: 807.1489. Anal. calcd $(\%)$ for $\mathrm{C}_{70} \mathrm{H}_{51} \mathrm{BF}_{24} \mathrm{FeIrPS}$ : C, 50.34; H, 3.08; S, 1.92; found: C, 50.27; H, 3.06; S, 1.89 .

[Ir(cod)(8)]BArF: Yield $114.9 \mathrm{mg}(91 \%) .{ }^{31} \mathrm{P}$ NMR (162 MHz, $\left.\mathrm{CDCl}_{3}\right), \delta: 6.6$ (s); ${ }^{1} \mathrm{H}$ NMR (400 MHz, $\mathrm{CDCl}_{3}$ ), $\delta: 1.62$ (m, 3H, $\left.\mathrm{CH}_{2}, \mathrm{cod}\right), 1.78$ (m, 2H, $\mathrm{CH}_{2}, \mathrm{cod}$ ), 2.28 (m, $3 \mathrm{H}, \mathrm{CH}_{2}$, cod and $\left.\mathrm{CH}_{2}-\mathrm{S}\right), 2.46\left(\mathrm{~m}, 1 \mathrm{H}, \mathrm{CH}_{2}\right.$, cod, and $\left.\mathrm{CH}_{2}-\mathrm{S}\right), 3.71(\mathrm{~m}, 1 \mathrm{H}, \mathrm{CH}=$, $\mathrm{Cp}), 3.86(\mathrm{~m}, 1 \mathrm{H}, \mathrm{CH}=, \operatorname{cod}), 4.04(\mathrm{~m}, 1 \mathrm{H}, \mathrm{CH}=, \mathrm{cod}), 4.26(\mathrm{~m}, 1 \mathrm{H}, \mathrm{CH}=, \mathrm{cod}), 4.51$ (m, 1H, CH=, Cp), 4.59 (s, 1H, CH=, Cp), 4.69 (s, 5H, CH=, Cp), 4.70 (m, 1H, CH=, cod), 7.4-7.9 (b, 29H, CH=); ${ }^{13} \mathrm{C} \mathrm{NMR} \mathrm{(100} \mathrm{MHz,} \mathrm{CDCl}_{3}$ ), $\delta: 27.8$ (b, $\left.\mathrm{CH}_{2}, \mathrm{cod}\right), 29.7$ (b, $\left.\mathrm{CH}_{2}, \operatorname{cod}\right), 30.2$ (b, $\left.\mathrm{CH}_{2}, \mathrm{cod}\right), 31.4\left(\mathrm{CH}_{2}-\mathrm{S}\right), 33.4\left(\mathrm{CH}_{2}-\mathrm{S}\right), 64.3\left(\mathrm{~d},{ }^{1} J_{\mathrm{C}-\mathrm{P}}=64.2 \mathrm{~Hz}\right.$, $\mathrm{C}, \mathrm{Cp}), 68.7\left(\mathrm{~d}, J_{\mathrm{C}-\mathrm{P}}=6.4 \mathrm{~Hz}, \mathrm{CH}=, \mathrm{Cp}\right), 71.3(\mathrm{CH}=, \mathrm{Cp}$ and $\mathrm{CH}=, \mathrm{cod}), 73.8(\mathrm{CH}=$, $\operatorname{cod}), 76.3\left(\mathrm{~d}, J_{\mathrm{C}-\mathrm{P}}=5.9 \mathrm{~Hz}, \mathrm{CH}=, \mathrm{Cp}\right), 84.9\left(\mathrm{~d},{ }^{2} J_{\mathrm{C}-\mathrm{P}}=20.4 \mathrm{~Hz}, \mathrm{C}, \mathrm{Cp}\right), 90.9\left(\mathrm{~d}, J_{\mathrm{C}-\mathrm{P}}=\right.$ $10.8 \mathrm{~Hz}, \mathrm{CH}=, \operatorname{cod}), 95.0$ (d, $\left.J_{\mathrm{C}-\mathrm{P}}=12.2 \mathrm{~Hz}, \mathrm{CH}=, \operatorname{cod}\right), 117.4\left(\mathrm{~b}, \mathrm{CH}=, \mathrm{BAr}_{\mathrm{F}}\right), 120.4-$ 134.2 (aromatic carbons), 134.8 (b, CH=, BAr $), 161.7$ (q, ${ }^{1} J_{\mathrm{C}-\mathrm{B}}=49.4 \mathrm{~Hz}, \mathrm{C}-\mathrm{B}, \mathrm{BAr}_{\mathrm{F}}$ ). TOF-MS (ESI+): $\mathrm{m} / \mathrm{z}=843.1487$, calcd. for $\mathrm{C}_{73} \mathrm{H}_{51} \mathrm{BF}_{24}$ FeIrPS $\left[\mathrm{M}-\mathrm{BAr}{ }_{\mathrm{F}}\right]^{+}:$843.1489. 
Anal. calcd (\%) for $\mathrm{C}_{73} \mathrm{H}_{51} \mathrm{BF}_{24} \mathrm{FeIrPS}$ : C, 51.39; H, 3.01; S, 1.88; found: C, 51.33; H, 2.99; S, 1.85. Major isomer (66\%): ${ }^{31} \mathrm{P}$ NMR (162 MHz, $\left.\mathrm{CDCl}_{3}, 228 \mathrm{~K}\right), \delta: 6.7$ (s); ${ }^{1} \mathrm{H}$ NMR (400 MHz, $\left.\mathrm{CDCl}_{3}, 228 \mathrm{~K}\right), \delta: 1.5$ - 2.5 (b, 8H, $\left.\mathrm{CH}_{2}, \mathrm{cod}\right), 3.64$ (m, 2H, CH=, cod), $3.70\left(\mathrm{~d}, 1 \mathrm{H},{ }^{2} J_{\mathrm{H}-\mathrm{H}}=12.0 \mathrm{~Hz}, \mathrm{CH}_{2}\right), 3.88(\mathrm{~m}, 1 \mathrm{H}, \mathrm{CH}=, \operatorname{cod}), 3.99(\mathrm{~b}, 1 \mathrm{H}, \mathrm{CH}=$, $\mathrm{Cp}), 4.17\left(\mathrm{~d}, 1 \mathrm{H},{ }^{2} \mathrm{~J}_{\mathrm{H}-\mathrm{H}}=12.0 \mathrm{~Hz}, \mathrm{CH}_{2}\right), 4.50(\mathrm{~b}, 1 \mathrm{H}, \mathrm{CH}=, \mathrm{Cp}), 4.60(\mathrm{~m}, 1 \mathrm{H}, \mathrm{CH}=, \mathrm{Cp})$, 4.63 (b, 1H, $\mathrm{CH}=$, cod), 4.67 (s, 5H, $\mathrm{CH}=, \mathrm{Cp}), 6.6-8.5$ (m, 29H, $\mathrm{CH}=)$. Minor isomer (33\%): ${ }^{31} \mathrm{P}$ NMR (162 MHz, $\left.\mathrm{CDCl}_{3}, 228 \mathrm{~K}\right), \delta: 8.8$ (s); ${ }^{1} \mathrm{H}$ NMR (400 MHz, $\mathrm{CDCl}_{3}, 228$ $\mathrm{K}), \delta: 1.5-2.7\left(\mathrm{~b}, 8 \mathrm{H}, \mathrm{CH}_{2}, \mathrm{cod}\right), 3.28\left(\mathrm{~d}, 1 \mathrm{H},{ }^{2} \mathrm{~J}_{\mathrm{H}-\mathrm{H}}=12.0 \mathrm{~Hz}, \mathrm{CH}_{2}\right), 3.64(\mathrm{~m}, 2 \mathrm{H}, \mathrm{CH}=$, cod), $3.94(\mathrm{~m}, 1 \mathrm{H}, \mathrm{CH}=, \mathrm{cod}), 4.20$ (b, 1H, CH=, Cp), 4.45 (b, 1H, CH=, Cp), 4.60 (b, $1 \mathrm{H}, \mathrm{CH}=, \mathrm{Cp}), 4.73(\mathrm{~s}, 5 \mathrm{H}, \mathrm{CH}=, \mathrm{Cp}), 4.88(\mathrm{~m}, 1 \mathrm{H}, \mathrm{CH}=, \operatorname{cod}), 6.6-8.5(\mathrm{~m}, 29 \mathrm{H}, \mathrm{CH}=)$.

[Ir(cod)(9)]BArF: Yield $113.4 \mathrm{mg}(91 \%) .{ }^{31} \mathrm{P} \mathrm{NMR}\left(162 \mathrm{MHz}, \mathrm{CDCl}_{3}\right), \delta: 5.9$ (s); ${ }^{1} \mathrm{H}$ NMR (400 MHz, $\mathrm{CDCl}_{3}$ ), $\delta: 1.76$ (m, 2H, $\left.\mathrm{CH}_{2}, \mathrm{cod}\right), 2.06$ (m, 1H, $\mathrm{CH}_{2}, \mathrm{cod}$ ), 2.20 (s, 3H, $\left.\mathrm{CH}_{3}\right), 2.29$ (m, 3H, $\left.\mathrm{CH}_{2}, \mathrm{cod}\right), 2.41$ (m, 1H, $\left.\mathrm{CH}_{2}, \mathrm{cod}\right), 2.56$ (m, 1H, $\left.\mathrm{CH}_{2}, \mathrm{cod}\right)$, $2.78\left(\mathrm{~s}, 3 \mathrm{H}, \mathrm{CH}_{3}\right), 3.42\left(\mathrm{~d}, 1 \mathrm{H},{ }^{2} J_{\mathrm{H}-\mathrm{H}}=13.2 \mathrm{~Hz}, \mathrm{CH}_{2}\right), 3.70(\mathrm{~m}, 1 \mathrm{H}, \mathrm{CH}=, \mathrm{cod}), 3.83(\mathrm{~m}$, $1 \mathrm{H}, \mathrm{CH}=$, cod), $3.92(\mathrm{~m}, 2 \mathrm{H}, \mathrm{CH}=$, cod and $\mathrm{Cp}), 3.99\left(\mathrm{~d}, 1 \mathrm{H},{ }^{2} J_{\mathrm{H}-\mathrm{H}}=13.2 \mathrm{~Hz}, \mathrm{CH}_{2}\right)$, $4.51(\mathrm{~m}, 2 \mathrm{H}, \mathrm{CH}=$, cod and $\mathrm{Cp}), 4.65(\mathrm{~s}, 6 \mathrm{H}, \mathrm{CH}=, \mathrm{Cp}), 7.1-7.8(\mathrm{~b}, 25 \mathrm{H}, \mathrm{CH}=) ;{ }^{13} \mathrm{C}$ NMR (100 MHz, $\left.\mathrm{CDCl}_{3}\right)$, \&: $22.9\left(\mathrm{CH}_{3}\right), 23.1\left(\mathrm{CH}_{3}\right), 27.9$ (b, $\left.\mathrm{CH}_{2}, \mathrm{cod}\right), 30.6$ (b, $\mathrm{CH}_{2}$, cod), $30.8\left(\mathrm{~d}, J_{\mathrm{C}-\mathrm{P}}=2.3 \mathrm{~Hz}, \mathrm{CH}_{2}\right.$, cod), 33.9 (d, $J_{\mathrm{C}-\mathrm{P}}=4.6 \mathrm{~Hz}, \mathrm{CH}_{2}$, cod), 35.2 (b, $\mathrm{CH}_{2-}$ S), $64.1\left(\mathrm{~d},{ }^{1} J_{\mathrm{C}-\mathrm{P}}=63.9 \mathrm{~Hz}, \mathrm{C}, \mathrm{Cp}\right), 68.5(\mathrm{CH}=, \operatorname{cod}), 68.7\left(\mathrm{~d}, J_{\mathrm{C}-\mathrm{P}}=6.1 \mathrm{~Hz}, \mathrm{CH}=, \mathrm{Cp}\right)$, $71.2(\mathrm{CH}=, \mathrm{Cp}), 73.0(\mathrm{CH}=, \operatorname{cod}), 73.9\left(\mathrm{~d}, J_{\mathrm{C}-\mathrm{P}}=2.1 \mathrm{~Hz}, \mathrm{CH}=, \mathrm{Cp}\right), 76.3\left(\mathrm{~d}, J_{\mathrm{C}-\mathrm{P}}=7.8\right.$ $\mathrm{Hz}, \mathrm{CH}=, \mathrm{Cp}), 85.3\left(\mathrm{~d},{ }^{2} J_{\mathrm{C}-\mathrm{P}}=16.0 \mathrm{~Hz}, \mathrm{C}, \mathrm{Cp}\right), 89.9$ (d, $\left.J_{\mathrm{C}-\mathrm{P}}=12.2 \mathrm{~Hz}, \mathrm{CH}=, \operatorname{cod}\right), 94.2$ $\left(\mathrm{d}, J_{\mathrm{C}-\mathrm{P}}=11.4 \mathrm{~Hz}, \mathrm{CH}=, \mathrm{cod}\right), 117.4$ (b, $\mathrm{CH}=, \mathrm{BAr}_{\mathrm{F}}$ ), 120.4-134.0 (aromatic carbons), 134.7 (b, $\left.\mathrm{CH}=, \mathrm{BAr}_{\mathrm{F}}\right), 140.3(\mathrm{C}), 142.0(\mathrm{C}), 161.6$ (q, $\left.{ }^{1} J_{\mathrm{C}-\mathrm{B}}=49.6 \mathrm{~Hz}, \mathrm{C}-\mathrm{B}, \mathrm{BAr}_{\mathrm{F}}\right)$. TOF-MS (ESI+): $\mathrm{m} / \mathrm{z}=821.1642$, calcd. for $\mathrm{C}_{71} \mathrm{H}_{53} \mathrm{BF}_{24} \mathrm{FeIrPS}\left[\mathrm{M}-\mathrm{BAr}_{\mathrm{F}}\right]^{+}:$821.1645. Anal. calcd (\%) for $\mathrm{C}_{71} \mathrm{H}_{53} \mathrm{BF}_{24}$ FeIrPS: C, 50.64; H, 3.17; S, 1.90; found: C, 50.61; H, $3.16 ; \mathrm{S}, 1.88$.

[Ir(cod)(10)]BArF: Yield 108.0 mg (90\%). ${ }^{31} \mathrm{P}$ NMR (162 MHz, $\left.\mathrm{CDCl}_{3}\right), \delta: 25.0$ (s); ${ }^{1} \mathrm{H}$ NMR (400 MHz, $\mathrm{CDCl}_{3}$ ), $\delta: 1.09$ (s, 9H, $\left.\mathrm{CH}_{3},{ }^{t} \mathrm{Bu}\right), 1.68\left(\mathrm{~m}, 1 \mathrm{H}, \mathrm{CH}_{2}, \mathrm{cod}\right), 1.84$ (m, 1H, $\left.\mathrm{CH}_{2}, \mathrm{cod}\right), 2.16$ (m, 2H, $\mathrm{CH}_{2}$, cod), 2.32 (m, 1H, $\left.\mathrm{CH}_{2}, \operatorname{cod}\right), 2.42$ (m, 1H, $\mathrm{CH}_{2}$, 
cod), $2.52\left(\mathrm{~m}, 2 \mathrm{H}, \mathrm{CH}_{2}\right.$, cod), 3.79 (m, 1H, $\mathrm{CH}=$, cod), 4.32 (s, 5H, CH=, Cp), 4.49 (b, $1 \mathrm{H}, \mathrm{CH}=, \mathrm{Cp}), 4.60(\mathrm{~m}, 1 \mathrm{H}, \mathrm{CH}=, \mathrm{cod}), 4.79(\mathrm{~m}, 1 \mathrm{H}, \mathrm{CH}=, \mathrm{cod}), 4.95(\mathrm{~b}, 1 \mathrm{H}, \mathrm{CH}=$, $\mathrm{Cp}), 5.21(\mathrm{~s}, 1 \mathrm{H}, \mathrm{CH}=, \mathrm{Cp}), 5.54(\mathrm{~s}, 1 \mathrm{H}, \mathrm{CH}=, \mathrm{cod}), 7.3-7.8(\mathrm{~m}, 22 \mathrm{H}, \mathrm{CH}=)$ ) ${ }^{13} \mathrm{C} \mathrm{NMR}$ $\left(100 \mathrm{MHz} \mathrm{CDCl}_{3}\right), \delta: 27.4\left(\mathrm{~d}, J_{\mathrm{C}-\mathrm{P}}=2.3 \mathrm{~Hz}, \mathrm{CH}_{2}, \operatorname{cod}\right), 29.1\left(\mathrm{~d}, J_{\mathrm{C}-\mathrm{P}}=2.2 \mathrm{~Hz}, \mathrm{CH}_{2}\right.$, cod), $31.0\left(\mathrm{CH}_{3},{ }^{t} \mathrm{Bu}\right), 33.2$ (b, $\left.\mathrm{CH}_{2}, \operatorname{cod}\right), 35.5$ (d, $\left.J_{\mathrm{C}-\mathrm{P}}=4.5 \mathrm{~Hz}, \mathrm{CH}_{2}, \operatorname{cod}\right), 61.8(\mathrm{C}$, $\left.{ }^{t} \mathrm{Bu}\right), 70.8(\mathrm{CH}=, \mathrm{Cp}), 71.7(\mathrm{CH}=, \operatorname{cod}), 72.7(\mathrm{CH}=, \mathrm{Cp}), 73.2(\mathrm{CH}=, \operatorname{cod}), 73.6\left(\mathrm{~d}, J_{\mathrm{C}-\mathrm{P}}\right.$ $=9.1 \mathrm{~Hz}, \mathrm{CH}=, \mathrm{Cp}), 79.2\left(\mathrm{~d},{ }^{1} J_{\mathrm{C}-\mathrm{P}}=62.1 \mathrm{~Hz}, \mathrm{C}, \mathrm{Cp}\right), 80.2\left(\mathrm{~d}, J_{\mathrm{C}-\mathrm{P}}=14.5 \mathrm{~Hz}, \mathrm{CH}=, \mathrm{Cp}\right)$, $85.2\left(\mathrm{~d},{ }^{2} J_{\mathrm{C}-\mathrm{P}}=26.4 \mathrm{~Hz}, \mathrm{C}, \mathrm{Cp}\right), 86.4\left(\mathrm{~d}, J_{\mathrm{C}-\mathrm{P}}=14.5 \mathrm{~Hz}, \mathrm{CH}=, \operatorname{cod}\right), 94.3\left(\mathrm{~d}, J_{\mathrm{C}-\mathrm{P}}=9.9\right.$ $\mathrm{Hz}, \mathrm{CH}=$, cod), 117.4 (b, $\mathrm{CH}=, \mathrm{BAr}_{\mathrm{F}}$ ), 120.4-133.1 (aromatic carbons), 134.7 (b, $\mathrm{CH}=$, $\left.\mathrm{BAr}_{\mathrm{F}}\right), 161.7\left(\mathrm{q}, J_{\mathrm{C}-\mathrm{B}}=49.4 \mathrm{~Hz}, \mathrm{C}-\mathrm{B}, \mathrm{BAr}_{\mathrm{F}}\right)$. TOF-MS (ESI+): m/z = 759.1484, calcd. for $\mathrm{C}_{66} \mathrm{H}_{51} \mathrm{BF}_{24} \mathrm{FeIrPS}$ [M-BArF] ${ }^{+}$: 759.1489. Anal. calcd (\%) for $\mathrm{C}_{66} \mathrm{H}_{51} \mathrm{BF}_{24} \mathrm{FeIrPS}$ : C, 48.87; H, 3.17; S, 1.98; found: C, 48.81; H, 3.15; S, 1.94 .

[Ir(cod)(11)]BArF: Yield $111.2 \mathrm{mg}(90 \%) .{ }^{31} \mathrm{P}$ NMR (162 MHz, $\left.\mathrm{CDCl}_{3}\right), \delta: 3.9$ (s); ${ }^{1} \mathrm{H}$ NMR (400 MHz, $\left.\mathrm{CDCl}_{3}\right), \delta: 0.71\left(\mathrm{~d}, 3 \mathrm{H},{ }^{3} \mathrm{~J}_{\mathrm{H}-\mathrm{H}}=6.8 \mathrm{~Hz}, \mathrm{CH}_{3}\right), 1.69\left(\mathrm{~m}, 2 \mathrm{H}, \mathrm{CH}_{2}\right.$, cod), 2.01 (m, 1H, $\mathrm{CH}_{2}$, cod), 2.17 (m, 1H, $\left.\mathrm{CH}_{2}, \mathrm{cod}\right), 2.42$ (m, 2H, $\left.\mathrm{CH}_{2}, \mathrm{cod}\right), 2.46$ (m, $\left.1 \mathrm{H}, \mathrm{CH}_{2}, \operatorname{cod}\right), 2.56\left(\mathrm{~m}, 1 \mathrm{H}, \mathrm{CH}_{2}, \operatorname{cod}\right), 3.48(\mathrm{~m}, 1 \mathrm{H}, \mathrm{CH}=, \operatorname{cod}), 3.54(\mathrm{~m}, 1 \mathrm{H}, \mathrm{CH}=$, cod), 4.11 (s, 1H, CH=, Cp), 4.32 (m, 1H, CH=, cod), 4.50 (s, 7H, CH=, Cp), 4.61 (q, $\left.1 \mathrm{H},{ }^{3} J_{\mathrm{H}-\mathrm{H}}=6.8 \mathrm{~Hz}, \mathrm{CH}\right), 4.72(\mathrm{~m}, 2 \mathrm{H}, \mathrm{CH}=, \mathrm{cod}), 7.5-7.8(\mathrm{~b}, 22 \mathrm{H}, \mathrm{CH}=) ;{ }^{13} \mathrm{C} \mathrm{NMR}$ (100 MHz, $\left.\mathrm{CDCl}_{3}\right), \delta: 22.9\left(\mathrm{CH}_{2}, \operatorname{cod}\right), 29.7\left(\mathrm{CH}_{2}, \operatorname{cod}\right), 31.0\left(\mathrm{CH}_{2}, \operatorname{cod}\right), 34.9\left(\mathrm{CH}_{2}\right.$, cod), $47.7(\mathrm{CH}), 63.5\left(\mathrm{~d},{ }^{1} J_{\mathrm{C}-\mathrm{P}}=61.5 \mathrm{~Hz}, \mathrm{C}, \mathrm{Cp}\right), 69.1$ (d, $\left.J_{\mathrm{C}-\mathrm{P}}=6.9 \mathrm{~Hz}, \mathrm{CH}=, \mathrm{Cp}\right), 70.9$ $(\mathrm{CH}=, \operatorname{cod}), 71.5(\mathrm{CH}=, \mathrm{Cp}), 72.1(\mathrm{CH}=, \operatorname{cod}), 74.1(\mathrm{CH}=, \operatorname{cod}), 74.5\left(\mathrm{~d}, J_{\mathrm{C}-\mathrm{P}}=6.1 \mathrm{~Hz}\right.$, $\mathrm{CH}=, \mathrm{Cp}), 90.8\left(\mathrm{~d},{ }^{2} J_{\mathrm{C}-\mathrm{P}}=16.7 \mathrm{~Hz}, \mathrm{C}, \mathrm{Cp}\right), 93.4\left(\mathrm{~d}, J_{\mathrm{C}-\mathrm{P}}=10.6 \mathrm{~Hz}, \mathrm{CH}=, \operatorname{cod}\right), 93.8(\mathrm{~d}$, $\left.J_{\mathrm{C}-\mathrm{P}}=10.6 \mathrm{~Hz}, \mathrm{CH}=, \mathrm{cod}\right), 117.4$ (b, $\mathrm{CH}=, \mathrm{BAr}_{\mathrm{F}}$ ), 120.4-134.2 (aromatic carbons), 134.7 (b, CH=, BAr $)$, $135.0(\mathrm{CH}=, \mathrm{Cp}), 161.7$ (q, $\left.\left.{ }^{1} J_{\mathrm{C}-\mathrm{B}}=49.8 \mathrm{~Hz}, \mathrm{C}-\mathrm{B}, \mathrm{BAr}\right)_{\mathrm{F}}\right)$. TOFMS (ESI+): $\mathrm{m} / \mathrm{z}=807.1488$, calcd. for $\mathrm{C}_{70} \mathrm{H}_{51} \mathrm{BF}_{24} \mathrm{FeIrPS}\left[\mathrm{M}-\mathrm{BAr}_{\mathrm{F}}\right]^{+}:$807.1489. Anal. calcd $(\%)$ for $\mathrm{C}_{70} \mathrm{H}_{51} \mathrm{BF}_{24}$ FeIrPS: C, 50.34; H, 3.08; S, 1.92; found: C, 50.31; H, 3.06; S, 1.90 .

[Ir(cod)(12)]BArF: Yield 111.9 mg (89\%). TOF-MS (ESI+): $\mathrm{m} / \mathrm{z}=835.1801$, calcd. for $\mathrm{C}_{72} \mathrm{H}_{55} \mathrm{BF}_{24} \mathrm{FeIrPS}$ [M-BAr $]^{+}$: 835.1802. Anal. calcd (\%) for $\mathrm{C}_{72} \mathrm{H}_{55} \mathrm{BF}_{24} \mathrm{FeIrPS}$ : C, 
50.93; H, 3.26; S, 1.89; found: C, 50.88; H, 3.24; S, 1.84 . Major isomer $(85 \%):{ }^{31} \mathrm{P}$ NMR (162 MHz, $\left.\mathrm{CDCl}_{3}\right), \delta: 3.0$ (s); ${ }^{1} \mathrm{H}$ NMR (400 MHz, $\left.\mathrm{CDCl}_{3}\right), \delta: 0.71$ (d, 3H, ${ }^{3} \mathrm{~J}_{\mathrm{H}-\mathrm{H}}$ $\left.=6.8 \mathrm{~Hz}, \mathrm{CH}_{3}\right), 1.83\left(\mathrm{~m}, 2 \mathrm{H}, \mathrm{CH}_{2}, \operatorname{cod}\right), 2.10\left(\mathrm{~m}, 1 \mathrm{H}, \mathrm{CH}_{2}, \operatorname{cod}\right), 2.30\left(\mathrm{~m}, 1 \mathrm{H}, \mathrm{CH}_{2}\right.$, cod), 2.39 (m, 2H, $\left.\mathrm{CH}_{2}, \operatorname{cod}\right), 2.46$ (m, 1H, $\mathrm{CH}_{2}$, cod), 2.57 (m, 1H, $\left.\mathrm{CH}_{2}, \mathrm{cod}\right), 2.62$ (s, $\left.3 \mathrm{H}, \mathrm{CH}_{3}-\mathrm{Ar}\right), 2.79$ (s, 3H, $\left.\mathrm{CH}_{3}-\mathrm{Ar}\right), 3.36(\mathrm{~m}, 1 \mathrm{H}, \mathrm{CH}=, \mathrm{cod}), 3.58(\mathrm{~m}, 1 \mathrm{H}, \mathrm{CH}=, \mathrm{cod})$, $4.00(\mathrm{~m}, 1 \mathrm{H}, \mathrm{CH}=, \mathrm{cod}), 4.11(\mathrm{~b}, 1 \mathrm{H}, \mathrm{CH}=, \mathrm{Cp}), 4.52$ (s, 6H, CH=, Cp), $4.62(\mathrm{~m}, 2 \mathrm{H}$, $\mathrm{CH}$ and $\mathrm{CH}=, \mathrm{Cp}), 4.64(\mathrm{~m}, 1 \mathrm{H}, \mathrm{CH}=, \mathrm{cod}), 7.2-7.7(\mathrm{~m}, 25 \mathrm{H}, \mathrm{CH}=) ;{ }^{13} \mathrm{C}$ NMR $(100$ $\left.\mathrm{MHz}, \mathrm{CDCl}_{3}\right), \delta: 21.2\left(\mathrm{CH}_{3}\right), 23.1\left(\mathrm{CH}_{3}-\mathrm{Ar}\right), 24.8\left(\mathrm{CH}_{3}-\mathrm{Ar}\right), 26.8\left(\mathrm{CH}_{2}, \operatorname{cod}\right), 29.8$ $\left(\mathrm{CH}_{2}, \mathrm{cod}\right), 31.2\left(\mathrm{CH}_{2}, \operatorname{cod}\right), 34.8\left(\mathrm{~d}, J_{\mathrm{C}-\mathrm{P}}=3.2 \mathrm{~Hz}, \mathrm{CH}_{2}, \mathrm{cod}\right), 47.0(\mathrm{CH}), 63.8\left(\mathrm{~d},{ }^{1} J_{\mathrm{C}-\mathrm{P}}\right.$ $=61.5 \mathrm{~Hz}, \mathrm{C}, \mathrm{Cp}), 69.0\left(\mathrm{~d}, J_{\mathrm{C}-\mathrm{P}}=6.9 \mathrm{~Hz}, \mathrm{CH}=, \mathrm{Cp}\right), 69.7(\mathrm{CH}=, \operatorname{cod}), 70.3(\mathrm{CH}=, \mathrm{cod})$, $71.4(\mathrm{CH}=, \mathrm{Cp}), 74.3\left(\mathrm{~d}, J_{\mathrm{C}-\mathrm{P}}=6.9 \mathrm{~Hz}, \mathrm{CH}=, \mathrm{Cp}\right), 74.8\left(\mathrm{~d}, J_{\mathrm{C}-\mathrm{P}}=3.8 \mathrm{~Hz}, \mathrm{CH}=, \mathrm{Cp}\right)$, $90.8\left(\mathrm{~d},{ }^{2} J_{\mathrm{C}-\mathrm{P}}=16.8 \mathrm{~Hz}, \mathrm{C}, \mathrm{Cp}\right), 92.6\left(\mathrm{~d}, J_{\mathrm{C}-\mathrm{P}}=11.4 \mathrm{~Hz}, \mathrm{CH}=, \operatorname{cod}\right), 94.2\left(\mathrm{~d}, J_{\mathrm{C}-\mathrm{P}}=10.6\right.$ $\mathrm{Hz}, \mathrm{CH}=, \mathrm{cod}), 117.4$ (b, $\mathrm{CH}=, \mathrm{BAr}_{\mathrm{F}}$ ), 120.4-134.7 (aromatic carbons), 134.7 (b, $\mathrm{CH}=$, $\mathrm{BAr}_{\mathrm{F}}$ ), 140.4-143.9 (aromatic carbons), 161.9 (q, ${ }^{1} J_{\mathrm{C}-\mathrm{B}}=49.4 \mathrm{~Hz}, \mathrm{C}-\mathrm{B}, \mathrm{BAr}{ }_{\mathrm{F}}$ ). Minor isomer (15\%): ${ }^{31} \mathrm{P} \mathrm{NMR}\left(162 \mathrm{MHz}, \mathrm{CDCl}_{3}\right), \delta: 5.7 \mathrm{ppm}(\mathrm{s}) ;{ }^{1} \mathrm{H} \mathrm{NMR}(400 \mathrm{MHz}$, $\left.\mathrm{CDCl}_{3}\right), \delta: 1.35\left(\mathrm{~d}, 3 \mathrm{H},{ }^{3} \mathrm{~J}_{\mathrm{H}-\mathrm{H}}=6.8 \mathrm{~Hz}, \mathrm{CH}_{3}\right), 1.92\left(\mathrm{~m}, 1 \mathrm{H}, \mathrm{CH}_{2}, \mathrm{cod}\right), 2.11(\mathrm{~m}, 1 \mathrm{H}$, $\mathrm{CH}_{2}, \operatorname{cod}$ ), 2.23 (s, 3H, $\mathrm{CH}_{3}-\mathrm{Ar}$ ), 2.2 - 2.6 (m, 6H, $\left.\mathrm{CH}_{2}, \mathrm{cod}\right), 2.74$ (s, 3H, $\mathrm{CH}_{3}-\mathrm{Ar}$ ), $3.63(\mathrm{~m}, 2 \mathrm{H}, \mathrm{CH}$ and $\mathrm{CH}=$, cod), $3.75(\mathrm{~m}, 2 \mathrm{H}, \mathrm{CH}=, \mathrm{cod}), 3.93(\mathrm{~m}, 1 \mathrm{H}, \mathrm{CH}=, \mathrm{Cp}), 4.52$ (b, 1H, CH=, cod), $4.56(\mathrm{~m}, 1 \mathrm{H}, \mathrm{CH}=, \mathrm{Cp}), 4.66$ (s, 5H, CH=, Cp), $4.73(\mathrm{~m}, 1 \mathrm{H}, \mathrm{CH}=$, $\mathrm{Cp})$, 7.2-7.7 (m, 25H, CH=); ${ }^{13} \mathrm{C} \mathrm{NMR}\left(100 \mathrm{MHz}, \mathrm{CDCl}_{3}\right), \delta: 17.1\left(\mathrm{CH}_{3}\right), 22.8\left(\mathrm{CH}_{3}-\right.$ Ar), $23.7\left(\mathrm{CH}_{3}-\mathrm{Ar}\right), 27.6\left(\mathrm{CH}_{2}, \operatorname{cod}\right), 29.6\left(\mathrm{CH}_{2}, \mathrm{cod}\right), 34.3\left(\mathrm{~d}, J_{\mathrm{C}-\mathrm{P}}=3.3 \mathrm{~Hz}, \mathrm{CH}_{2}, \mathrm{cod}\right)$, $42.8(\mathrm{CH}), 64.8\left(\mathrm{~d},{ }^{1} J_{\mathrm{C}-\mathrm{P}}=60.8 \mathrm{~Hz}, \mathrm{C}, \mathrm{Cp}\right), 69.3(\mathrm{~b}, \mathrm{CH}=, \mathrm{Cp}), 71.1(\mathrm{CH}=, \mathrm{Cp}), 72.3$ $(\mathrm{CH}=, \mathrm{cod}), 74.4\left(\mathrm{~d}, J_{\mathrm{C}-\mathrm{P}}=5.8 \mathrm{~Hz}, \mathrm{CH}=, \mathrm{Cp}\right), 75.2\left(\mathrm{~d}, J_{\mathrm{C}-\mathrm{P}}=3.2 \mathrm{~Hz}, \mathrm{CH}=, \mathrm{Cp}\right), 89.3(\mathrm{~d}$, $\left.{ }^{2} J_{\mathrm{C}-\mathrm{P}}=19.1 \mathrm{~Hz}, \mathrm{C}, \mathrm{Cp}\right), 90.1\left(\mathrm{~d}, J_{\mathrm{C}-\mathrm{P}}=10.4 \mathrm{~Hz}, \mathrm{CH}=, \operatorname{cod}\right), 95.7\left(\mathrm{~d}, J_{\mathrm{C}-\mathrm{P}}=14.3 \mathrm{~Hz}\right.$, $\mathrm{CH}=, \operatorname{cod}), 134.7\left(\mathrm{~b}, \mathrm{CH}=, \mathrm{BAr}_{\mathrm{F}}\right), 140.4-143.9$ (aromatic carbons), 161.7 (q, ${ }^{1} J_{\mathrm{C}-\mathrm{B}}=$ 49.4 Hz, C-B, BArF). 


\section{General procedure for the hydrogenation of olefins}

The alkene $(0.5 \mathrm{mmol})$ and $\mathrm{Ir}$ complex $(1 \mathrm{~mol} \%)$ were dissolved in $\mathrm{CH}_{2} \mathrm{Cl}_{2}(2 \mathrm{~mL})$ in a high-pressure autoclave, which was purged four times with hydrogen. Then, it was pressurized to the desired pressure. After the desired reaction time, the autoclave was depressurized and the solvent evaporated off. The residue was dissolved in $\operatorname{Et}_{2} \mathrm{O}(1.5$ ml) and filtered through a short Celite plug. The enantiomeric excess was determined by chiral GC or chiral HPLC and conversions were determined by ${ }^{1} \mathrm{H}$ NMR. The enantiomeric excesses of hydrogenated products from S1-S5, ${ }^{10 a} \mathbf{S 6},{ }^{37} \mathbf{S 7},{ }^{10 a} \mathbf{S 8}-\mathbf{S 9}, 5^{\circ}$ S10 $,{ }^{38} \mathbf{S} 11,5^{\circ} \mathbf{S 1 2},{ }^{39} \mathbf{S} 13,{ }^{40} \mathbf{S} 14,5^{\mathrm{i}} \mathbf{S 1 5 - S 1 6}, 5^{\mathrm{v}} \mathbf{S 1 7},{ }^{26 \mathrm{a}} \mathbf{S 1 8},{ }^{27 \mathrm{~b}} \mathbf{S 1 9 - S 2 3},{ }^{28 \mathrm{a}} \mathbf{S 2 4},{ }^{41} \mathbf{S 2 5},{ }^{10 \mathrm{a}}$ S26-S32, ${ }^{42} \mathbf{S 3 3},{ }^{29 \mathrm{a}} \mathbf{S 3 4},{ }^{28 \mathrm{a}}$ were determined using the conditions described previously.

\section{Acknowledgements}

We thank the Spanish Government for providing grant CTQ2013-40568, the Catalan Government for grant 2014SGR670, and the ICREA Foundation for providing M. Diéguez and O. Pàmies with financial support through the ICREA Academia awards. We also thank the Centre National de la Recherche Scientifique, the Institut Universitaire de France, and the Laboratoire Européen Associé "Laboratoire TransPyrénéen: de la Molécule aux Matériaux” for additional support.

\section{References}

${ }^{1}$ a) Comprehensive Asymmetric Catalysis; Eds.: Jacobsen, E. N.; Pfaltz, A.; Yamamoto; H.; Springer-Verlag: Berlin, 1999; b) Catalytic Asymmetric Synthesis; 3rd Edition; Ed.: Ojima, I.; John Wiley \& Sons, Inc.: Hoboken, 2010; c) Asymmetric Catalysis in Industrial Scale: Challenges, Approaches and Solutions; 2nd Ed; Eds.: Blaser, H. U.; Federsel, H.-J.; Wiley: Weinheim, Germany, 2010; d) Asymmetric Catalysis in Organic Synthesis; Ed.: Noyori; R.: Wiley: New York, 1994; e) Busacca, C. A.; Fandrick, D. R.; Song, J. J.; Senanayakl, C. H. Adv. Synth. Catal. 2011, 353, 1825. 
2 a) Brown, J. M. in Comprehensive Asymmetric Catalysis; Eds.: Jacobsen, E. N.; Pfaltz, A.; Yamamoto, H.; Springer-Verlag: Berlin, 1999; Vol. I, pp 121-182. b) Wang, D.-S.; Chen, Q.-A.; Lu, S.-M.; Zhou, Y.-G. Chem. Rev. 2012, 112, 2557. d) Knowles, W. S.; Noyori, R. Acc. Chem. Res. 2007, 40, 1238.

${ }^{3}$ For reviews, see: a) Cui, X.; Burgess, K. Chem. Rev. 2005, 105, 3272. b) Källström, K.; Munslow, I.; Andersson, P. G. Chem. Eur. J. 2006, 12, 3194. c) Roseblade, S. J.; Pfaltz, A. Acc. Chem. Res. 2007, 40, 1402. d) Church, T. L.; Andersson, P. G. Coord. Chem. Rev. 2008, 252, 513. e) Pàmies, O.; Andersson, P. G.; Diéguez, M. Chem. Eur. J. 2010, 16, 14232. f) Woodmansee, D. H.; Pfaltz, A. Chem. Commun. 2011, 47, 7912. g) Zhu, Y.; Burgess, K. Acc. Chem. Res. 2012, 45, 1623. h) Verendel, J. J.; Pàmies, O.; Diéguez, M.; Andersson, P. G. Chem. Rev. 2014, 114, 2130.

${ }^{4}$ Lightfoot, A.; Schnider, P.; Pfaltz, A. Angew. Chem. Int. Ed. 1998, 37, 2897.

${ }^{5}$ See, for instance: a) Blankenstein, J.; Pfaltz, A. Angew. Chem. Int. Ed. 2001, 40, 4445. b) Hou, D.-R.; Reibenspies, J.; Colacot, T. J.; Burgess, K. Chem. Eur. J. 2001, 7, 5391. c) Menges, F.; Pfaltz, A. Adv. Synth. Catal. 2002, 344, 40. d) Perry, M. C.; Cui, X.; Powell, M. T.; Hou, D.-R.; Reibenspies, J. H.; Burgess, K. J. Am. Chem. Soc. 2003, 125, 113. e) Tang, W.; Wang, W.; Zhang, X. Angew. Chem. Int. Ed. 2003, 42, 943. f) Liu, D.; Tang, W.; Zhang, X. Org. Lett. 2004, 6, 513. g) McIntyre, S.; Hörmann, E.; Menges, F.; Smidt, S. P.; Pfaltz, A. Adv. Synth. Catal. 2005, 347, 282. h) Trifonova, A.; Diesen, J. S.; Andersson, P. G. Chem. Eur. J. 2006, 12, 2318. i) Lu, S.-M.; Bolm, C. Angew. Chem. Int. Ed. 2008, 47, 8920. j) Diéguez, M.; Mazuela, J.; Pàmies, O.; Verendel, J. J.; Andersson, P. G. J. Am. Chem. Soc. 2008, 130, 7208. k) Diéguez, M.; Pàmies, O.; Verendel, J. J.; Andersson, P. G. Chem. Commun. 2008, 3888. 1) Engman, M.; Cheruku, P.; Tolstoy, P.; Bergquist, J.; Völker, S. F.; Andersson, P. G. Adv. Synth. Catal. 2009, 351, 375. m) Zhao, J.; Burgess, K. J. Am. Chem. Soc. 2009, 131, 13236. n) Mazuela, J.; Verendel, J. J.; Coll, M.; Schäffner, B.; Börner, A.; Andersson, P. G.; Pàmies, O.; Diéguez, M. J. Am. Chem. Soc. 2009, 131, 12344. o) Lu, W.-J.; Chen, Y.W.; Hou, X.-L. Adv. Synth. Catal. 2010, 352, 103. p) Zhang, Y.; Han, Z.; Li, F.; Ding, 
K.; Zhang, A. Chem. Commun. 2010, 46, 156. q) Verendel, J. J.; Zhou, T.; Li, J.-Q.; Paptchikhine, A.; Lebedev, O.; Andersson, P. G. J. Am. Chem. Soc. 2010, 132, 8880. r) Mazuela, J.; Norrby, P.-O.; Andersson, P. G.; Pàmies, O.; Diéguez, M. J. Am. Chem. Soc. 2011, 133, 13634. s) Franzke, A.; Pfaltz, A. Chem. Eur. J. 2011, 17, 4131. t) Shang, J.; Han, Z.; Li, Y.; Wang, X.; Ding, K. Chem. Commun. 2012, 48, 5172. u) Wang, X.; Han, Z.; Wang, Z.; Ding, K. Angew. Chem. Int. Ed. 2012, 51, 936. v) Verendel, J. J.; Li, J.-Q.; Quan, X.; Peters, B.; Zhou, T.; Gautun, O. R.; Govender, T.; Andersson, P. G. Chem. Eur. J. 2012, 18, 6507. w) Mazuela, J.; Pàmies, O.; Diéguez, M. Eur. J. Inorg. Chem. 2013, 2139. x) Khumsubdee, S.; Fan, Y.; Burgess, K. J. Org. Chem. 2013, 78, 9969. y) Zhu, Y.; Burgess, K. RSC Advances 2012, 2, 4728. z) Müller, M.-A.; Pfaltz, A. Angew. Chem Int. Ed. 2014, 53, 8668.

${ }^{6}$ a) Bunlaksananusorn, T.; Polborn, K.; Knochel, P. Angew. Chem. Int. Ed. 2003, 42, 3941. b) Drury III, W. J.; Zimmermann, N.; Keenan, M.; Hayashi, M.; Kaiser, S.; Goddard, R.; Pfaltz, A. Angew. Chem. Int. Ed. 2004, 43, 70. c) Bell, S.; Wüstenberg, B.; Kaiser, S.; Menges, F.; Netscher, T.; Pfaltz, A. Science 2006, 311, 642-. d) Kaiser, S.; Smidt, S. P.; Pfaltz, A. Angew. Chem. Int. Ed. 2006, 45, 5194. e) Margalef, J.; Lega, M.; Ruffo, F.; Pàmies, O.; Diéguez, M. Tetrahedron: Asymmetry 2012, 23, 945. f) Woodmansee, D. H.; Müller, M.-A.; Tröndlin, L.; Hörmann, E.; Pfaltz, A. Chem. Eur. J. 2012, 18, 13780. g) Mazuela, J.; Pàmies, O.; Diéguez, M. Adv. Synth. Catal. 2013, 355, 2569. h) Schumacher, A.; Bernasconi, M.; Pfaltz, A. Angew. Chem. Int. Ed. 2013, $52,7422$.

${ }^{7}$ Rageot, D.; Woodmansee, D. H.; Pugin, B.; Pfaltz, A. Angew. Chem. Int. Ed. 2011, $50,9598$.

8 a) Hedberg, C.; Källström, K.; Brandt, P.; Hansen, L. K.; Andersson, P. G. J. Am. Chem. Soc. 2006, 128, 2995. b) Cheruku, P.; Paptchikhine, A.; Church, T. L.; Andersson, P. G. J. Am. Chem. Soc. 2009, 131, 8285. c) Tolstoy, P.; Engman, M.; Paptchikhine, A.; Bergquist, J.; Church, T. L.; Leung, A. W.-M.; Andersson, P. G. J. 
Am. Chem. Soc. 2009, 131, 8855. d) Verendel, J. J.; Li, J.-Q.; Quan, X.; Peters, B.; Zhou, T.; Gautun, O. R.; Govender, T.; Andersson, P. G. Chem. Eur. J. 2012, 18, 6507.

${ }^{9}$ Mazuela, J.; Pàmies, O.; Diéguez, M. ChemCatChem 2013, 5, 2410.

${ }^{10}$ a) Källström, K.; Hedberg, C.; Brandt, P.; Bayer, A.; Andersson, P. G. J. Am. Chem. Soc. 2004, 126, 14308. b) Mazuela, J.; Paptchikhine, A.; Pàmies, O.; Andersson, P. G.; Diéguez, M. Chem. Eur. J. 2010, 16, 4567.

11 a) Coll, M.; Pàmies, O.; Diéguez, M. Chem. Commun. 2011, 47, 9215. b) Coll, M.; Pàmies, O.; Diéguez, M. Adv. Synth. Catal. 2013, 355, 143. c) Margalef, J.; Caldentey, X.; Karlsson, E. A.; Coll, M.; Mazuela, J.; Pàmies, O.; Diéguez, M.; Pericàs, M. A. Chem. Eur. J. 2014, 20, 12201.

12 a) Manoury, E.; Fossey, J. S.; Aït-Haddou, H.; Daran, J.-C.; Balavoine, G. G. A. Organometallics 2000, 19, 3736. b) Lopez Cortes, J. G.; Ramon, O.. Vincendeau, S.; Serra, D.; Lamy, F.; Daran, J.-C.; Manoury, E.; Gouygou, M. Eur. J. Inorg. Chem., 2006, 5148. c) Debono, N.; Labande, A.; Manoury, E.; Daran, J.-C.; Poli, R. Organometallics 2010, 29, 1879. d) Wei, M.-M.; García-Melchor, M.; Lledós, A.; Audin, C.; Daran, J.-C.; Poli, R.; Deydier, E.; Manoury, E. Organometallics 2012, 31, 6669. e) Malacea, R.; Daran, J.-C.; Poli, R.; Manoury, E. Tetrahedron: Asymmetry, 2013, 24, 612 .

${ }^{13}$ For recent reviews about chiral ferrocenyl ligands in asymmetric catalysis: a) Colacot, T. J. Chem. Rev. 2003, 103, 3101. b) Atkinson, R. C. J.; Gibson, V. C.; Long, N. J. Chem. Soc. Rev. 2004, 33, 313. c) Gomez Arrayas, R.; Adrio, J.; Carretero, J. C. Angew. Chem. Int. Ed. 2006, 45, 7674. d) Drusan, M.; Sebesta, R. Tetrahedron 2014, 70, 759.

${ }^{14}$ a) Li, X.; Li, Q.; Wu, X.; Gao, Y.; Xu, D.; Kong, L. Tetrahedron:Asymmetry 2007, 18, 629. b) Metallinos, C.; Van Belle, L. J. Organomet. Chem. 2011, 696, 141. c) Gschwend, B.; Pugin, B.; Bertogg, A.; Pfaltz, A. Chem. Eur. J. 2009, 15, 12993. d) Co, T. T.; Kim, T.-J. Chem. Commun. 2006, 3537. e) Gazić Smilović, I.; Casas-Arcé, E.; Roseblade, S. J.; Nettekoven, U.; Zanotti-Gerosa, A.; Kovačevič, M.; Časar, Z. Angew. Chem. Int. Ed. 2012, 51, 1014. 
These ligands have been applied in allylic substitution reactions, methoxycarbonylation and hydrogenation of alkynes, imines and ketones. See for instance: a) Routaboul, L.; Vincendeau, S.; Daran, J.-C.; Manoury, E. Tetrahedron: Asymmetry 2005, 16, 2685. b) Diab, L.; Gouygou, M.; Manoury, E.; Kalck, P.; Urrutigoity, M. Tetrahedron Lett. 2008, 49, 5186. c) Le Roux, E.; Malacea, R.; Manoury, E.; Poli, R.; Gonsalvi, L.; Peruzzini, M. Adv. Synth. Catal. 2007, 349, 309. d) Kozinets, E. M.; Silantyev, G. A.; Belkova, N. V.; Shubina, E. S.; Poli, R.; Manoury, E. Russ. Chem. Bull. 2013, 62, 751.

16 Fesulphos ligand $\mathbf{1 0}$ has been successfully used in several asymmetric metalcatalyzed reactions (i.e. 1,3-dipolar cycloadditions, aza-Diels Alder and Mannich reactions, allylic substitution, ...). See for instance: a) Hernando, E.; Arrayas, R. G.; Carretero, J. C. Chem. Commun. 2012, 48, 9622. b) Filippone, S.; Maroto, E. E.; Martin-Domenech, A.; Suarez, M.; Martin, N. Nature Chem. 2009, 1, 578. c) Hernandez-Toribio, J.; Arrayas, R. G.; Carretero, J. C. J. Am. Chem. Soc. 2008, 130, 16150. d) Cabrera, S.; Arrayas, R. G.; Carretero, J. C. J. Am. Chem. Soc. 2005, 127, 16394. e) Cabrera, S.; Arrayas, R. G.; Carretero, J. C. Angew. Chem. Int. Ed. 2004, 43, 3944. f) Mancheno, O. G.; Arrayas, R. G.; Carretero, J. C. J. Am. Chem. Soc. 2004, 126, 456. g) Priego, J.; Mancheno, O. G.; Cabrera, S.; Arrayas, R. G.; Llamas, T.; Carretero, J. C. Chem. Commun. 2002, 2512.

17 These ligands have been applied in hydrosilylation, [3+2] cycloaddition and allylic substitution reactions. See: a) Zeng, W.; Zhou, Y.-G. Tetrahedron Lett. 2007, 48, 4619. b) Tu, T.; Zhou, Y.-G.; Hou, X.-L.; Dai, L.-X.; Dong, X.-C.; Yu, Y.-H.; Sun, J. Organometallics 2003, 22, 1255. c) Nishibayashi, Y.; Segawa, K.; Singh, J. D.; Fukuzawa, S.; Ohe, K.; Uemura, S. Organometallics 1996, 15, 370.

18 Malacea, R., Manoury, E., Routaboul, L., Daran, J.-C., Poli, R., Dunne, J. P., Withwood, A. C., Godard, C., Duckett, S. B. Eur. J. Inorg. Chem., 2006, 1803.

19 a) Malacea, R.; Daran, J.-C.; Duckett, S. B.; Dunne, J. P.; Manoury, E.; Poli, R.; Withwood, A. C. Dalton Trans. 2006, 3350. c) Malacea, R.; Daran, J.-C.; Duckett, S. 
B.; Dunne, J. P.; Manoury, E.; Poli, R.; Withwood, A. C. Dalton Trans. 2006, 3350. d)

Diab, L.; Daran, J.-C.; Gouygou, M.; Manoury, E.; Urrutigoïty, M. Acta Cryst. Sect. C 2007, C63, m586. e) Malacea, R.; Routaboul, L.; Manoury, E.; Daran, J.-C.; Poli, R. J. Organomet. Chem. 2008, 693,1469. f) Malacea, R.; Manoury, E.; Daran, J.-C.; Poli, R. J. Mol. Struct. 2008, 890, 249. g) Kozinets, E. M.; Koniev, O.; Fillipov, O. A.; Daran, J.-C.; Poli, R.; Shubina, E. S.; Belkova, N. V.; Manoury, E. Dalton Trans. 2012, 41, 11849.

${ }^{20}$ Schrems, M. G.; Neumann, E.; Pfaltz, A. Angew. Chem. Int. Ed. 2007, 46, 8274.

${ }^{21}$ For recent successful applications, see: a) Li, J.-Q.; Quan, X.; Andersson, P. G. Chem Eur. J. 2012, 18, 10609. b) Woodmansee, D. H.; Müller, M.-A.; Tröndlin, L.; Hörmann, E.; Pfaltz, A. Chem. Eur. J. 2012, 18, 13780.

${ }^{22}$ See for example: a) Judge, T. M.; Phillips, G.; Morris, J. K.; Lovasz, K. D.; Romines, K. R.; Luke, G. P.; Tulinsky, J.; Tustin, J. M.; Chrusciel, R. A.; Dolak, L. A.; Mizsak, S. A.; Watt, W.; Morris, J.; Velde, S. L. V.; Strohbach, J. W.; Gammill, R. B. J. Am. Chem. Soc. 1997, 119, 3627. b) Kraft, P.; Bajgrowicz, J. A.; Denis, C.; Frater, G. Angew. Chem. Int. Ed. 2000, 39, 2980. c) Sturm, T.; Weissensteiner, W.; Spindler, F. Adv. Synth. Catal. 2003, 345, 160. d) Henke, B. R. J. Med. Chem. 2004, 47, 4118. e) Saudan, L. A. Acc. Chem. Res. 2007, 40, 1309. f) Zanotti-Gerosa, A.; Kinney, W. A.; Grasa, G. A.; Medlock, J., Seger, A.; Ghosh, S.; Teleha, C. A.; Maryanoff, B. E. Tetrahedron: Asymmetry 2008, 19, 938.

23 a) Leutenegger, U.; Madin, A.; Pfaltz, A. Angew. Chem. Int. Ed. Engl. 1989, 28, 60. b) Yamada, T. M; Ohtsuka, Y.; Ikeno, T. Chem. Lett. 1998, 1129.

${ }^{24}$ a) Appella, D. H.; Moritani, Y.; Shintani, R.; Ferreira, E. M.; Buchwald, S. L. J. Am. Chem. Soc. 1999, 121, 9473. b) Hughes, G.; Kimura, M.; Buchwald, S. L. J. Am. Chem. Soc. 2003, 125, 11253. c) Tsuchiya, Y.; Kanazwa, Y.; Shiomo, T.; Kobayashi, K.; Nishiyama, H. Synlett 2004, 2493.

25 a) Lu, W.-J.; Chen, Y.-W.; Hou, X.-L. Angew. Chem., Int. Ed. 2008, 47, 10133. b) Maurer, F.; Huch, V.; Ullrich, A.; Kazmaier, U. J. Org. Chem. 2012, 77, 5139. 
${ }^{26}$ For successful application, see: a) Lu, W.-J.; Hou, X.-L. Adv. Synth. Catal. 2009, 351, 1224. b) Shang, J.; Han, Z.; Li, Y.; Wang, Z.; Ding, K. Chem. Commun. 2012, 48, 5172. ${ }^{27}$ For successful applications, see: a) Tian, F.; Yao, D.; Liu, Y.; Xie, F.; Zhang W. Adv. Synth. Catal. 2010, 352, 1841. b) Liu, X.; Han, Z.; Wang, Z.; Ding K. Angew. Chem. Int. Ed. 2014, 53, 1978. c) ref. 21a. d) ref. 21 b.

${ }^{28}$ For successful applications, see: a) Cheruku, P.; Diesen, J.; Andersson, P. G. J. Am. Chem. Soc. 2008, 130, 5595. b) ref. 10b.

${ }^{29}$ Only two catalytic systems have provided high enantioselectivities, see: a) Cheruku, P.; Gohil, S.; Andersson, P. G. Org. Lett. 2007, 9, 1659 (ee's up to 95\%). b) ref. 10b (ee's up to $82 \%$ ).

${ }^{30}$ Whittall, J.; Mbafor, W. F.; McCormack, P. J. Brit. UK Pat. Appl. 2005, GB 2410026 A 20050720.

31 Frisch, M. J.; Trucks, G. W.; Schlegel, H. B.; Scuseria, G. E.; Robb, M. A.; Cheeseman, J. R.; Scalmani, G. ; Barone, V.; Mennucci, B.; Petersson, G. A.; Nakatsuji, H.; Caricato, M.; Li, X.; Hratchian, H. P.; Izmaylov, A. F.; Bloino, J.; Zheng, G.; Sonnenberg, J. L.; Hada, M.; Ehara, M.; Toyota, K.; Fukuda, R.; Hasegawa, J.; Ishida, M.; Nakajima, T.; Honda, Y.; Kitao, O.; Nakai, H.; Vreven, T.; Montgomery, J. A.; Peralta, Jr., J. E.; Ogliaro, F.; Bearpark, M.; Heyd, J. J.; Brothers, E. ; Kudin, K. N.; Staroverov, V. N.; Kobayashi, R.; Normand, J.; Raghavachari, K.; Rendell, A.; Burant, J. C.; Iyengar, S. S.; Tomasi, J.; Cossi, M.; Rega, N.; Millam, J. M.; Klene, M.; Knox, J. E.; Cross, J. B.; Bakken, V.; Adamo, C.; Jaramillo, J.; Gomperts, R.; Stratmann, R. E.; Yazyev, O.; Austin, A. J.; Cammi, R; Pomelli, C.; Ochterski, J. W.; Martin, R. L.; Morokuma, K.; Zakrzewski, V. G.; Voth, G. A.; Salvador, P.; Dannenberg, J. J.; Dapprich, S.; Daniels, A. D.; Farkas, Ö.; Foresman, J. B.; Ortiz, J. V.; Cioslowski, J.; Fox, D. J.; Revision A.02 ed., Gaussian, Inc., Wallingford CT, 2009.

32 a) Lee, C.; Yang, W.; Parr, R. G. Phys. Rev. B 1988, 37, 785. b) Becke, A. D. J. Chem. Phys. 1993, 98, 5648.

${ }^{33}$ Hay, P. J.; Wadt, W. R. J. Chem. Phys. 1985, 82, 299. 
34 a) Hehre, W. J.; Ditchfield, R.; Pople, J. A. J. Chem. Phys. 1972, 56, 2257. b) Hariharan, P. C.; Pople, J. A. Theor. Chim. Acta 1973, 28, 213. c) Francl, M. M.; Pietro, W. J.; Hehre, W. J.; Binkley, J. S.; Gordon, M. S.; Defrees, D. J.; Pople, J. A. J. Chem. Phys. 1982, 77, 3654.

35 a) Miertus, S.; Tomasi, J. Chem. Phys. 1982, 65, 239. b) Mennucci, B.; Tomasi, J. J. Chem. Phys. 1997, 106, 5151. c) Cossi, M.; Barone, V.; Menucci, B.; Tomasi, J. Chem. Phys. Lett. 1998, 286, 253.

${ }^{36}$ a) Grimme, S.; Antony, J; Ehrlich, S.; Krieg, H. J. Chem. Phys. 2010, 132, 15410. b) Grimme, S.; Ehrlich, S.; Goerigk, L. J. Comput. Chem. 2011, 32, 1456.

${ }^{37}$ Woodmansee, D. H.; Müller, M.-A.; Neuburger, M. A. Pfaltz, Chem. Sci. 2010, 1, 72.

${ }^{38}$ Deasy, R. E.; Brossat, M.; Moody, T. S.; Maguire, A. R. Tetrahedron: Asymmetry 2011, 22, 47.

${ }^{39}$ Paptchikhine, A.; Cheruku, P.; Engman, M.; Andersson, P. G. Chem. Commun. 2009, 5996.

${ }^{40}$ Ganić, A.; Pfaltz, A. Chem. Eur. J. 2012, 18, 6724.

${ }^{41}$ Ohta, T.; Ikegami, H.; Miyake, T.; Takaya, H. J. Organomet. Chem. 1995, 502, 169.

${ }^{42}$ See Supporting Information for details. 


\section{Graphical Abstract}

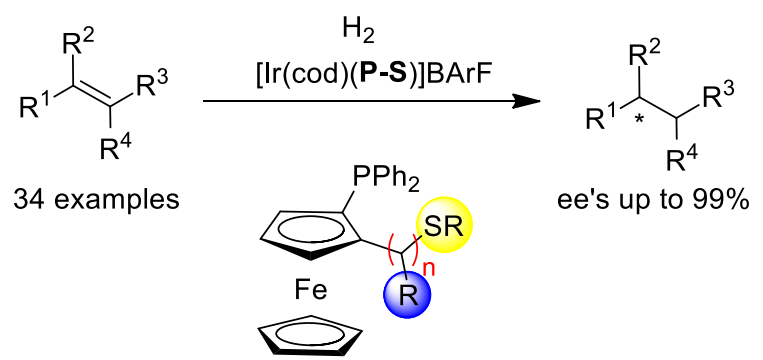

